\title{
Interval Entropy of Fuzzy Sets and the Application to Fuzzy Multiple Attribute Decision Making
}

\author{
Yiying Shi ${ }^{1,2}$ and Xuehai Yuan ${ }^{1,3}$ \\ ${ }^{1}$ School of Control Science and Engineering, Dalian University of Technology, Dalian 116024, China \\ ${ }^{2}$ School of Science, Shenyang Ligong University, Shenyang 110159, China \\ ${ }^{3}$ School of Science, Dalian University of Technology, Panjin Campus, Panjin 124221, China \\ Correspondence should be addressed to Yiying Shi; shiyiying98@163.com
}

Received 20 May 2015; Revised 27 August 2015; Accepted 2 September 2015

Academic Editor: Rita Gamberini

Copyright (C) 2015 Y. Shi and X. Yuan. This is an open access article distributed under the Creative Commons Attribution License, which permits unrestricted use, distribution, and reproduction in any medium, provided the original work is properly cited.

\begin{abstract}
A series of new concepts including interval entropy, interval similarity measure, interval distance measure, and interval inclusion measure of fuzzy sets are introduced. Meanwhile, some theorems and corollaries are proposed to show how these definitions can be deduced from each other. And then, based on interval entropy, a fuzzy multiple attribute decision making (FMADM) model is set up. In this model, interval entropy is used as the weight, by which the evaluation values of all alternatives can be obtained. Then all alternatives with respect to each criterion can be ranked as the order of the evaluation values. At last, a practical example is given to illustrate an application of the developed model and a comparative analysis is made.
\end{abstract}

\section{Introduction}

Multiple attribute decision making (MADM) problems existed in the economic, management, and various social fields. And it refers to making preference decisions over the available alternatives that are characterized by multiple attributes. But for practical needs and peoples' more profound understanding, there is much uncertain information included in the decision making process. How to handle the uncertain information is the issue that decision makers must be concerned about. In 1965, the fuzzy set theory was proposed by Zadeh, which provided effective methods to solve the issue. Several years later, Bellman and Zadeh [1] put forward a fuzzy model based on MADM method by combining fuzzy set and decision making. In the model, the attributes which cannot be defined exactly will be expressed as some proper fuzzy sets and converted into classical decision making problems by use of level sets. Because of its great flexibility and adaptability, the model has been widely viewed as the basis of fuzzy decision making. The FMADM method, proposed by Bass and Kwakernaak in 1977 [2], is regarded as the classical method in fuzzy decision making. In recent years, many new methods have been applied to
FMADM problems (such as hesitant fuzzy theory [3-6], TOPSIS method $[7,8]$, some operators [9-11], preference relations [12], and intuitionistic fuzzy decision making [13]). Hadi-Vencheh and Mirjaberi [14] developed an approach to solve MADM problems considering distances both to the positive ideal solution and to the negative ideal solution. Zhang and $\mathrm{Xu}$ [15] proposed an extended technique for order preference based on Pythagorean fuzzy set. In this approach, a score function based comparison method is proposed to identify the Pythagorean fuzzy positive ideal solution and the Pythagorean fuzzy negative ideal solution. And a distance measure is defined to calculate the distances between each alternative and the Pythagorean fuzzy positive ideal solution as well as the Pythagorean fuzzy negative ideal solution, respectively. And the comparative analysis had been made among these methods [16].

As Zimmermann [17] pointed out, the existing methods can be divided into two steps: the first is to determine the weights of all alternatives and then combine them into the evaluation values with fuzzy operator; the second is to rank the order of all alternatives according to the evaluation values. For MADM problems, when the weight of alternative is defined, results of decision making depended on the values 
of alternatives. And the alternative weights in MADM can be classified as subjective and objective alternative weights based on the information acquisition approach. The subjective alternative weights are determined by preference information on the alternatives given by the decision maker. The objective alternative weights are determined by the decision making matrix. In terms of determining objective alternative weights, one of the most famous approaches is the entropy method, which expresses the relative intensities of alternative importance to signify the average intrinsic information transmitted to the decision maker. Entropy concept was used in various scientific fields, especially in information theory, and used to refer to a general measure of uncertainty [18]. In MADM, the greater the value of the entropy corresponding to a special attribute is, which means the smaller attribute's weight, the less the discriminate power of that attribute in decision making process is. So far, a lot of literature pertaining to MADM analysis has been published using entropy weights, for instance, the cross entropy [19, 20], fuzzy entropy [21], Shannon entropy [22], maximizing fuzzy entropy [23], and sine entropy [24]. Jin et al. [25] proposed the interval value intuitionistic fuzzy continuous weighted entropy which generalizes intuitionistic fuzzy entropy measures defined by Szmidt and Kacprzyk on the basis of the continuous ordered weighted averaging operator. Zhang et al. [26] investigated the MADM problem with completely unknown attribute weights in the framework of interval value intuitionistic fuzzy sets. Using a new definition of interval value intuitionistic fuzzy entropy and some calculation methods for interval value intuitionistic fuzzy entropy, an entropy-based decision making method to solve interval value intuitionistic FMADM problems with completely unknown attribute weights is proposed. Besides the above results, there are also some papers focused on the relationships between entropy and other concepts (such as distance measure [27], similarity measure $[28,29]$, and inclusion measure [30-32]).

For the above methods, the common point is to use different entropies as weights. That is to say, the weight value is one specific number. But in practice, because of uncertainty of people's cognition, the data of the decision making processes cannot be measured precisely and there may be some other types of data, for instance, interval value data. In other words, the decision maker would prefer to express the point of view in this form rather than a real number because of the uncertainty and the lack of certain data, especially when data are known to lie within bounded variables, or when facing missing data, judgment data, and so forth. In MADM it is most probable that we confront such a case. So when the weight is an interval value, how to use entropy to represent it is worthy of discussion.

In this paper, according to the definition of fuzzy set entropy, the concept of interval entropy on fuzzy sets is proposed, and its application in MADM is introduced. There are four sections in the paper. Firstly, Section 2 is preliminary, and interval entropy of fuzzy set is proposed in Section 3. Secondly, the relationships among interval entropy, interval similarity measure, interval distance measure, and interval inclusion measure are discussed in Section 4. Finally, the FMADM analysis has been conducted in risk assessment of Taiwan railway reconstruction project in Section 5.

\section{Preliminary}

In this section, some definitions are introduced. Here, let $X$ be a set. The mapping $\mu_{A}: X \rightarrow[0,1]$ is called a fuzzy subset of $X$. Let $F(X)$ and $P(X)$ denote the class of all fuzzy sets and crisp sets over $X$, respectively.

Definition 1 (see [33]). A fuzzy number is defined as a fuzzy subset with the membership function $\mu_{A}(x)$, for any $x \in R$ satisfies the following properties:

(1) $A$ is a normal fuzzy set; that is, $\exists x \in R, \mu_{A}(x)=1$.

(2) $A$ is a convex fuzzy set; that is to say, for any $x_{1}, x_{2}$ and $\lambda \in[0,1]$,

$$
\mu_{A}\left(\lambda x_{1}+(1-\lambda) x_{2}\right) \geq \min \left(\mu_{A}\left(x_{1}\right), \mu_{A}\left(x_{2}\right)\right) .
$$

(3) The support of $A$ is bounded; that is to say, the set $\operatorname{Supp}(A)=\left\{x \in R \mid \mu_{A}(x)>0\right\}$ is bounded.

Definition 2 (see [7]). The fuzzy number $A$ is called the trapezoidal fuzzy number and denoted by $[a, b, c, d]$, where the membership function can be expressed as the following:

$$
\mu_{A}(x)= \begin{cases}\frac{x-a}{b-a} & a \leq x \leq b \\ 1 & b \leq x \leq c \\ \frac{d-x}{d-c} & c \leq x \leq d \\ 0 & \text { else. }\end{cases}
$$

If $b=c$, then $A$ is called the triangular fuzzy number and denoted by $[a, m, d]$. Therefore, a triangular fuzzy number is a special case of the trapezoidal fuzzy number.

Definition 3 (see $[7,34,35]$ ). For $\alpha \in(0,1]$, the $\alpha$-cut set $A_{\alpha}$ of $A$ is a classic set defined as

$$
A_{\alpha}=\left\{x \in R \mid \mu_{A}(x) \geq \alpha\right\}
$$

If $A$ is the trapezoidal fuzzy number, then $A_{\alpha}$ can be denoted by

$$
A_{\alpha}= \begin{cases}{[b \alpha+a(1-\alpha), c \alpha+d(1-\alpha)]} & \alpha \in(0,1) \\ {[b, c]} & \alpha=1\end{cases}
$$

Let $D([0,1])=\left\{\left[a^{-}, a^{+}\right] \mid 0 \leq a^{-} \leq a^{+} \leq 1\right\}$ be the interval value set; we set [25]

(1) $\left[a^{-}, a^{+}\right] \leq\left[b^{-}, b^{+}\right] \Leftrightarrow a^{-} \leq b^{-}, a^{+} \leq b^{+}$;

(2) $\left[a^{-}, a^{+}\right]=\left[b^{-}, b^{+}\right] \Leftrightarrow a^{-}=b^{-}, a^{+}=b^{+}$;

(3) $\left[a^{-}, a^{+}\right]^{c}=\left[1-a^{+}, 1-a^{-}\right]$. 


\section{Interval Entropy of Fuzzy Sets}

In fact, entropy of fuzzy sets can be used to describe the general measure of fuzziness through the mapping between fuzzy numbers and real numbers on $[0,1]$, just like the process of the defuzzification, in which only one point is used to represent the fuzzy number. But, in many real life problems, the data of the decision making processes cannot be measured precisely. For instance, when the fuzziness of the fuzzy set can be expressed as a maximum of 0.8 and a minimum of 0.2 , how about it? It is necessary to extend the value of entropy from the number to interval value, and then the definition of interval entropy is proposed as follows.

Definition 4. A real function IE: $F(X) \rightarrow D([0,1]) A \mapsto$ $\operatorname{IE}(A)$ is called interval entropy on $F(X)$, if IE has the following properties:

(EP1) If $A \in P(X)$, then $\operatorname{IE}(A)=[0,0]$.

(EP2) If $\forall x \in X, \mu_{A}(x) \equiv 1 / 2$, then $\operatorname{IE}(A)=[1,1]$.

(EP3) If $\forall x \in X, \mu_{A_{2}}(x) \leq \mu_{A_{1}}(x) \leq 1 / 2$ or $\mu_{A_{2}}(x) \geq$ $\mu_{A_{1}}(x) \geq 1 / 2$, then

$$
\operatorname{IE}\left(A_{2}\right) \leq \operatorname{IE}\left(A_{1}\right)
$$

(EP4) $\operatorname{IE}(A)=\operatorname{IE}\left(A^{c}\right)$.

Remark 5. It can be concluded that if $\operatorname{IE}\left(A_{1}\right)=\left[a_{1}^{-}, a_{1}^{+}\right]$, $\operatorname{IE}\left(A_{2}\right)=\left[a_{2}^{-}, a_{2}^{+}\right] \in D([0,1])$, then we have

$$
\operatorname{IE}\left(A_{1}\right) \leq \operatorname{IE}\left(A_{2}\right) \Longleftrightarrow a_{1}^{-} \leq a_{2}^{-}, a_{1}^{+} \leq a_{2}^{+} .
$$

We can construct some interval entropy formulas based on Definition 4 as follows.

Let $X=\left\{x_{1}, x_{2}, \ldots, x_{n}\right\}$;

$$
\begin{aligned}
& \operatorname{IE}_{1}(A)=\left[\frac{1}{n} \sum_{i=1}^{n} 2\left(\mu_{A}\left(x_{i}\right) \wedge \mu_{A}^{c}\left(x_{i}\right)\right), \frac{1}{n}\right. \\
& \left.\cdot \sum_{i=1}^{n} 4\left(\mu_{A}\left(x_{i}\right) \cdot \mu_{A}^{c}\left(x_{i}\right)\right)\right] \text {; } \\
& \mathrm{IE}_{2}(A)=\left[\frac{1}{n} \sum_{i=1}^{n} 4\left(\left(\mu_{A}\left(x_{i}\right)\right)^{2} \wedge\left(\mu_{A}^{c}\left(x_{i}\right)\right)^{2}\right), \frac{1}{n}\right. \\
& \left.\cdot \sum_{i=1}^{n} \frac{\mu_{A}\left(x_{i}\right) \wedge \mu_{A}^{c}\left(x_{i}\right)}{\mu_{A}\left(x_{i}\right) \vee \mu_{A}^{c}\left(x_{i}\right)}\right] \\
& \operatorname{IE}_{3}(A)=\left[\frac{1}{n} \sum_{i=1}^{n} \frac{\mu_{A}\left(x_{i}\right) \wedge \mu_{A}^{c}\left(x_{i}\right)}{\mu_{A}\left(x_{i}\right) \vee \mu_{A}^{c}\left(x_{i}\right)}, \frac{1}{n}\right. \\
& \left.\cdot \sum_{i=1}^{n} 2\left(\mu_{A}\left(x_{i}\right) \wedge \mu_{A}^{c}\left(x_{i}\right)\right)\right] \text {; }
\end{aligned}
$$

$$
\begin{gathered}
\mathrm{IE}_{4}(A)=\left[\frac{1}{n} \sum_{i=1}^{n} 2\left(\mu_{A}\left(x_{i}\right) \wedge \mu_{A}^{c}\left(x_{i}\right)\right), \frac{1}{n}\right. \\
\left.\cdot \sum_{i=1}^{n} \frac{2\left(\mu_{A}\left(x_{i}\right) \cdot \mu_{A}^{c}\left(x_{i}\right)\right)}{\left(\mu_{A}\left(x_{i}\right)\right)^{2}+\left(\mu_{A}^{c}\left(x_{i}\right)\right)^{2}}\right] ; \\
\mathrm{IE}_{5}(A)=\left[\frac{1}{n} \sum_{i=1}^{n} \frac{2\left(\mu_{A}\left(x_{i}\right) \cdot \mu_{A}^{c}\left(x_{i}\right)\right)}{2}+\left(\mu_{A}^{c}\left(x_{i}\right)\right)^{2}, \frac{1}{n}\right. \\
\left.\cdot \sum_{i=1}^{n} 4\left(\mu_{A}\left(x_{i}\right) \cdot \mu_{A}^{c}\left(x_{i}\right)\right)\right] ; \\
\operatorname{IE}_{6}(A)=\left[\frac{1}{n} \sum_{i=1}^{n}\left(\frac{\mu_{A}\left(x_{i}\right) \wedge \mu_{A}^{c}\left(x_{i}\right)}{\mu_{A}\left(x_{i}\right) \vee \mu_{A}^{c}\left(x_{i}\right)}\right)^{p}, \frac{1}{n}\right. \\
\left.\cdot \sum_{i=1}^{n}\left(\frac{\mu_{A}\left(x_{i}\right) \wedge \mu_{A}^{c}\left(x_{i}\right)}{\mu_{A}\left(x_{i}\right) \vee \mu_{A}^{c}\left(x_{i}\right)}\right)^{1 / p}\right](p \geq 1) .
\end{gathered}
$$

If $\mu_{A}(x)$ can be integrated over the considered interval $[a, b]$, then

$$
\begin{aligned}
& \operatorname{IE}_{7}(A)=\left[\frac{1}{b-a} \int_{a}^{b} 2\left(\mu_{A}(x) \wedge \mu_{A}^{c}(x)\right) d x, \frac{1}{b-a}\right. \\
& \left.\cdot \int_{a}^{b} 4\left(\mu_{A}(x) \cdot \mu_{A}^{c}(x)\right) d x\right] \text {, } \\
& \mathrm{IE}_{8}(A)=\left[\frac{1}{b-a}\right. \\
& \cdot \int_{a}^{b} 4\left(\left(\mu_{A}(x)\right)^{2} \wedge\left(\mu_{A}^{c}(x)\right)^{2}\right) d x, \frac{1}{b-a} \\
& \left.\cdot \int_{a}^{b} \frac{\mu_{A}(x) \wedge \mu_{A}^{c}(x)}{\mu_{A}(x) \vee \mu_{A}^{c}(x)} d x\right] \text {; } \\
& \operatorname{IE}_{9}(A)=\left[\frac{1}{b-a} \int_{a}^{b} \frac{\mu_{A}(x) \wedge \mu_{A}^{c}(x)}{\mu_{A}(x) \vee \mu_{A}^{c}(x)} d x, \frac{1}{b-a}\right. \\
& \left.\cdot \int_{a}^{b} 2\left(\mu_{A}(x) \wedge \mu_{A}^{c}(x)\right) d x\right] \\
& \operatorname{IE}_{10}(A)=\left[\frac{1}{b-a} \int_{a}^{b} 2\left(\mu_{A}(x) \wedge \mu_{A}^{c}(x)\right) d x, \frac{1}{b-a}\right. \\
& \left.\cdot \int_{a}^{b} \frac{2\left(\mu_{A}(x) \cdot \mu_{A}^{c}(x)\right)}{\left(\mu_{A}(x)\right)^{2}+\left(\mu_{A}^{c}(x)\right)^{2}} d x\right] \\
& \operatorname{IE}_{11}(A)=\left[\frac{1}{b-a} \int_{a}^{b} \frac{2\left(\mu_{A}(x) \cdot \mu_{A}^{c}(x)\right)}{\left(\mu_{A}(x)\right)^{2}+\left(\mu_{A}^{c}(x)\right)^{2}} d x, \frac{1}{b-a}\right. \\
& \left.\int_{a}^{b} 4\left(\mu_{A}(x) \cdot \mu_{A}^{c}(x)\right) d x\right]
\end{aligned}
$$




$$
\begin{gathered}
\operatorname{IE}_{12}(A)=\left[\frac{1}{b-a} \int_{a}^{b}\left(\frac{\mu_{A}(x) \wedge \mu_{A}^{c}(x)}{\mu_{A}(x) \vee \mu_{A}^{c}(x)}\right)^{p} d x, \frac{1}{b-a}\right. \\
\left.\cdot \int_{a}^{b}\left(\frac{\mu_{A}(x) \wedge \mu_{A}^{c}(x)}{\mu_{A}(x) \vee \mu_{A}^{c}(x)}\right)^{1 / p} d x\right] \quad(p \geq 1) .
\end{gathered}
$$

The following theorem shows that the above formulas are all interval entropies.

Theorem 6. $I E_{i}(A, B)(i=1,2, \ldots, 12)$ are interval entropies.

Proof. When $i=7$, by (7), we have

$$
\begin{gathered}
\operatorname{IE}_{7}(A)=\left[\frac{1}{b-a} \int_{a}^{b} 2\left(\mu_{A}(x) \wedge \mu_{A}^{c}(x)\right) d x, \frac{1}{b-a}\right. \\
\left.\cdot \int_{a}^{b} 4\left(\mu_{A}(x) \cdot \mu_{A}^{c}(x)\right) d x\right] .
\end{gathered}
$$

Firstly, if $0 \leq \mu_{A}(x) \leq 1 / 2$, then $2\left(\mu_{A}(x) \wedge \mu_{A}^{c}(x)\right)=2 \mu_{A}(x)$ and $1 / 2 \leq \mu_{A}^{c}(x) \leq 1$, so

$$
2 \mu_{A}(x) \leq 4 \mu_{A}(x) \cdot \mu_{A}^{c}(x) \leq 1
$$

Namely,

$$
\begin{aligned}
0 & \leq \frac{1}{b-a} \int_{a}^{b} 2\left(\mu_{A}(x) \wedge \mu_{A}^{c}(x)\right) d x \\
& \leq \frac{1}{b-a} \int_{a}^{b} 4\left(\mu_{A}(x) \cdot \mu_{A}^{c}(x)\right) d x \leq 1 .
\end{aligned}
$$

So

$$
\begin{gathered}
\mathrm{IE}_{7}(A)=\left[\frac{1}{b-a} \int_{a}^{b} 2\left(\mu_{A}(x) \wedge \mu_{A}^{c}(x)\right) d x, \frac{1}{b-a}\right. \\
\left.\cdot \int_{a}^{b} 4\left(\mu_{A}(x) \cdot \mu_{A}^{c}(x)\right) d x\right] \in D([0,1]) .
\end{gathered}
$$

To (EP1): if $A \in P(X)$, then $\mu_{A}(x)=0$ or $\mu_{A}(x)=1$. So

$$
\begin{aligned}
2\left(\mu_{A}(x) \wedge \mu_{A}^{c}(x)\right) & =0, \\
4 \mu_{A}(x) \cdot \mu_{A}^{c}(x) & =0 .
\end{aligned}
$$

Namely, $\operatorname{IE}_{7}(A)=[0,0]$.

(EP2) If $\forall x \in X, \mu_{A}(x) \equiv 1 / 2$, then $2\left(\mu_{A}(x) \wedge \mu_{A}^{c}(x)\right)=1$, $4 \mu_{A}(x) \cdot \mu_{A}^{c}(x)=1$.

Namely, $\operatorname{IE}_{7}(A)=[1,1]$.
(EP3) If $\forall x \in X, \mu_{A_{2}}(x) \leq \mu_{A_{1}}(x) \leq 1 / 2$, then

$$
\begin{aligned}
& \mathrm{IE}_{7}\left(A_{1}\right)=\left[\frac{1}{b-a} \int_{a}^{b} 2\left(\mu_{A_{1}}(x) \wedge \mu_{A_{1}}^{c}(x)\right) d x, \frac{1}{b-a}\right. \\
& \left.\cdot \int_{a}^{b} 4\left(\mu_{A_{1}}(x) \cdot \mu_{A_{1}}^{c}(x)\right) d x\right]=\left[\frac{1}{b-a}\right. \\
& \cdot \int_{a}^{b} 2 \mu_{A_{1}}(x) d x, \frac{1}{b-a} \\
& \left.\cdot \int_{a}^{b} 4\left(\mu_{A_{1}}(x) \cdot \mu_{A_{1}}^{c}(x)\right) d x\right], \\
& \mathrm{IE}_{7}\left(A_{2}\right)=\left[\frac{1}{b-a} \int_{a}^{b} 2\left(\mu_{A_{2}}(x) \wedge \mu_{A_{2}}^{c}(x)\right) d x, \frac{1}{b-a}\right. \\
& \left.\cdot \int_{a}^{b} 4\left(\mu_{A_{2}}(x) \cdot \mu_{A_{2}}^{c}(x)\right) d x\right]=\left[\frac{1}{b-a}\right. \\
& \quad \cdot \int_{a}^{b} 2 \mu_{A_{2}}(x) d x, \frac{1}{b-a} \\
& \left.\cdot \int_{a}^{b} 4\left(\mu_{A_{2}}(x) \cdot \mu_{A_{2}}^{c}(x)\right) d x\right] .
\end{aligned}
$$

For $\mu_{A_{2}}(x) \leq \mu_{A_{1}}(x) \leq 1 / 2$, then we have

$$
\begin{gathered}
2 \mu_{A_{2}}(x) \leq 2 \mu_{A_{1}}(x), \\
4 \mu_{A_{2}}(x) \cdot \mu_{A_{2}}^{c}(x) \leq 4 \mu_{A_{1}}(x) \cdot \mu_{A_{1}}^{c}(x) .
\end{gathered}
$$

Namely,

$$
\operatorname{IE}_{7}\left(A_{2}\right) \leq \operatorname{IE}_{7}\left(A_{1}\right) \text {. }
$$

When $\mu_{A_{2}}(x) \geq \mu_{A_{1}}(x) \geq 1 / 2$, the proof is similar.

(EP4) It can be easily concluded that $\operatorname{IE}_{7}(A)=\operatorname{IE}_{7}\left(A^{c}\right)$.

The proofs for other cases are similar.

In particular, if $A$ is a trapezoidal fuzzy number and denoted bys $A=[a, b, c, d]$, we have

$$
\begin{aligned}
& \operatorname{IE}_{7}(A)=\left[\frac{b-a-c+d}{2(d-a)}, \frac{2(b-a-c+d)}{3(d-a)}\right] ; \\
& \mathrm{IE}_{8}(A) \\
& \quad=\left[\frac{b-a-c+d}{3(d-a)}, \frac{(2 \ln 2-1)(b-a-c+d)}{d-a}\right] ;
\end{aligned}
$$

$\mathrm{IE}_{9}(A)$

$$
=\left[\frac{(2 \ln 2-1)(b-a-c+d)}{d-a}, \frac{b-a-c+d}{2(d-a)}\right] ;
$$

$\mathrm{IE}_{10}(A)=\left[\frac{b-a-c+d}{2(d-a)}, \frac{(\pi-2)(b-a-c+d)}{2(d-a)}\right]$;

$\operatorname{IE}_{11}(A)$

$$
=\left[\frac{(\pi-2)(b-a-c+d)}{2(d-a)}, \frac{2(b-a-c+d)}{3(d-a)}\right] .
$$




\section{Property}

4.1. Other Definitions to Describe the Uncertainty. In fact, the conceptions to describe the general measure of uncertainty such as similarity measure, distance measure, and inclusion measure have also built the mapping between two fuzzy numbers and real numbers on $[0,1]$, in which only one point is used to represent two fuzzy numbers (see appendix). But when the values are interval, it is necessary to extend these conceptions to interval values, and, in this section, the definitions of interval similarity measure, interval distance measure, and interval inclusion measure are proposed as follows.

Definition 7. A real function IS: $F(X) \times F(X) \rightarrow D([0,1])$ is called interval similarity measure on $F(X)$ if IS satisfies the following properties:

(SP1) $\operatorname{IS}\left(A, A^{c}\right)=[0,0]$ if $A$ is a crisp set.

(SP2) $\operatorname{IS}(A, A)=[1,1]$.

(SP3) $\operatorname{IS}(A, B)=\operatorname{IS}(B, A)$.

(SP4) For all $A, B, C \in F(X)$, if $A \subseteq B \subseteq C$, then

$$
\begin{aligned}
& \operatorname{IS}(A, C) \leq \operatorname{IS}(A, B), \\
& \operatorname{IS}(A, C) \leq \operatorname{IS}(B, C) .
\end{aligned}
$$

We can construct some interval similarity measure formulas based on Definition 7 as follows.

For instance, let $X=\left\{x_{1}, x_{2}, \ldots, x_{n}\right\}$; we set

$$
\begin{aligned}
& \mathrm{IS}_{1}(A, B)=\left[\frac{1}{n} \sum_{i=1}^{n} \frac{\mu_{A}\left(x_{i}\right) \wedge \mu_{B}\left(x_{i}\right)}{\mu_{A}\left(x_{i}\right) \vee \mu_{B}\left(x_{i}\right)}, 1\right. \\
& \left.-\max _{x_{i} \in X}\left|\mu_{A}\left(x_{i}\right)-\mu_{B}\left(x_{i}\right)\right|\right] ; \\
& \mathrm{IS}_{2}(A, B)=\left[\frac{1}{n} \sum_{i=1}^{n} \frac{\mu_{A}\left(x_{i}\right) \wedge \mu_{B}\left(x_{i}\right) \vee \mu_{B}\left(x_{i}\right)}{\mu_{A}}, \frac{1}{n}\right. \\
& \cdot \sum_{i=1}^{n} \frac{2 \mu_{A}\left(x_{i}\right) \cdot \mu_{B}\left(x_{i}\right)}{\left.\left(\mu_{A}\left(x_{i}\right)\right)^{2}+\left(\mu_{B}\left(x_{i}\right)\right)^{2}\right] ;} \\
& \mathrm{IS}_{3}(A, B)=\left[\frac{1}{n} \sum_{i=1}^{n} \frac{\mu_{A}\left(x_{i}\right) \wedge \mu_{B}\left(x_{i}\right)}{\mu_{A}\left(x_{i}\right)}, 1-\frac{1}{n}\right. \\
& \left.\cdot \sum_{i=1}^{n}\left|\frac{\mu_{A}\left(x_{i}\right)-\mu_{B}\left(x_{i}\right)}{\mu_{A}\left(x_{i}\right)+\mu_{B}\left(x_{i}\right)}\right|\right] ; \\
& \operatorname{IS}_{4}(A, B)=\left[1-\frac{1}{n} \sum_{i=1}^{n}\left|\frac{\mu_{A}\left(x_{i}\right)-\mu_{B}\left(x_{i}\right)}{\mu_{A}\left(x_{i}\right)+\mu_{B}\left(x_{i}\right)}\right|, \frac{1}{n}\right. \\
& \left.\left(\mu_{A}\left(x_{i}\right)\right)^{2}+\left(\mu_{B}\left(x_{i}\right)\right)^{2}\right] ;
\end{aligned}
$$

$$
\begin{gathered}
\operatorname{IS}_{5}(A, B)=\left[\frac{1}{n^{p}} \sum_{i=1}^{n}\left(\frac{\mu_{A}\left(x_{i}\right) \wedge \mu_{B}\left(x_{i}\right)}{\mu_{A}\left(x_{i}\right) \vee \mu_{B}\left(x_{i}\right)}\right)^{p}, \frac{1}{n^{1 / p}}\right. \\
\left.\cdot \sum_{i=1}^{n}\left(\frac{\mu_{A}\left(x_{i}\right) \wedge \mu_{B}\left(x_{i}\right)}{\mu_{A}\left(x_{i}\right) \vee \mu_{B}\left(x_{i}\right)}\right)^{1 / p}\right] \quad(p \geq 1) .
\end{gathered}
$$

If $\mu_{A}(x)$ and $\mu_{B}(x)$ can be integrated over the considered interval $[a, b]$, then

$$
\begin{aligned}
& \operatorname{IS}_{6}(A, B)=\left[\frac{1}{b-a} \int_{a}^{b} \frac{\mu_{A}(x) \wedge \mu_{B}(x)}{\mu_{A}(x) \vee \mu_{B}(x)} d x, 1\right. \\
& \left.-\max _{x \in[a, b]}\left|\mu_{A}(x)-\mu_{B}(x)\right|\right] \text {; } \\
& \operatorname{IS}_{7}(A, B)=\left[\frac{1}{b-a} \int_{a}^{b} \frac{\mu_{A}(x) \wedge \mu_{B}(x)}{\mu_{A}(x) \vee \mu_{B}(x)} d x, \frac{1}{b-a}\right. \\
& \left.\cdot \int_{a}^{b} \frac{2 \mu_{A}(x) \cdot \mu_{B}(x)}{\left(\mu_{A}(x)\right)^{2}+\left(\mu_{B}(x)\right)^{2}} d x\right] \\
& \operatorname{IS}_{8}(A, B)=\left[\frac{1}{b-a} \int_{a}^{b} \frac{\mu_{A}(x) \wedge \mu_{B}(x)}{\mu_{A}(x) \vee \mu_{B}(x)} d x, 1-\frac{1}{b-a}\right. \\
& \left.\int_{a}^{b}\left|\frac{\mu_{A}(x)-\mu_{B}(x)}{\mu_{A}(x)+\mu_{B}(x)}\right| d x\right] \text {; } \\
& \mathrm{IS}_{9}(A, B)=\left[1-\frac{1}{b-a}\right. \\
& \int_{a}^{b}\left|\frac{\mu_{A}(x)-\mu_{B}(x)}{\mu_{A}(x)+\mu_{B}(x)}\right| d x, \frac{1}{b-a} \\
& \left.\cdot \int_{a}^{b} \frac{2 \mu_{A}(x) \cdot \mu_{B}(x)}{\left(\mu_{A}(x)\right)^{2}+\left(\mu_{B}(x)\right)^{2}} d x\right] \text {; } \\
& \mathrm{IS}_{10}(A, B)=\left[\frac{1}{(b-a)^{p}}\right. \\
& \cdot \int_{a}^{b}\left(\frac{\mu_{A}(x) \wedge \mu_{B}(x)}{\mu_{A}(x) \vee \mu_{B}(x)}\right)^{p} d x, \frac{1}{(b-a)^{1 / p}} \\
& \left.\cdot \int_{a}^{b}\left(\frac{\mu_{A}(x) \wedge \mu_{B}(x)}{\mu_{A}(x) \vee \mu_{B}(x)}\right)^{1 / p} d x\right] \quad(p \geq 1) .
\end{aligned}
$$

The following theorem shows that the above formulas are all interval similarity measures.

Theorem 8. $I S_{i}(A, B)(i=1,2, \ldots, 10)$ are interval similarity measures.

Proof. When $i=2$, we have

$$
\begin{gathered}
\mathrm{IS}_{2}(A, B)=\left[\frac{1}{n} \sum_{i=1}^{n} \frac{\mu_{A}\left(x_{i}\right) \wedge \mu_{B}\left(x_{i}\right)}{\mu_{A}\left(x_{i}\right) \vee \mu_{B}\left(x_{i}\right)}, \frac{1}{n}\right. \\
\left.\cdot \sum_{i=1}^{n} \frac{2 \mu_{A}\left(x_{i}\right) \cdot \mu_{B}\left(x_{i}\right)}{\left(\mu_{A}\left(x_{i}\right)\right)^{2}+\left(\mu_{B}\left(x_{i}\right)\right)^{2}}\right] .
\end{gathered}
$$


If $\mu_{A}\left(x_{i}\right) \leq \mu_{B}\left(x_{i}\right)$, then $\left(\mu_{A}\left(x_{i}\right) \wedge \mu_{B}\left(x_{i}\right)\right) /\left(\mu_{A}\left(x_{i}\right) \vee \mu_{B}\left(x_{i}\right)\right)=$ $\mu_{A}\left(x_{i}\right) / \mu_{B}\left(x_{i}\right)$,

$$
\begin{aligned}
& \frac{2 \mu_{A}\left(x_{i}\right) \cdot \mu_{B}\left(x_{i}\right)}{\left(\mu_{A}\left(x_{i}\right)\right)^{2}+\left(\mu_{B}\left(x_{i}\right)\right)^{2}}-\frac{\mu_{A}\left(x_{i}\right)}{\mu_{B}\left(x_{i}\right)} \\
& =\frac{\mu_{A}\left(x_{i}\right)\left[\left(\mu_{B}\left(x_{i}\right)\right)^{2}-\left(\mu_{A}\left(x_{i}\right)\right)^{2}\right]}{\left[\left(\mu_{A}\left(x_{i}\right)\right)^{2}+\left(\mu_{B}\left(x_{i}\right)\right)^{2}\right] \mu_{B}\left(x_{i}\right)} \geq 0 .
\end{aligned}
$$

Namely,

$$
\begin{aligned}
0 & \leq \frac{1}{n} \sum_{i=1}^{n} \frac{\mu_{A}\left(x_{i}\right) \wedge \mu_{B}\left(x_{i}\right)}{\mu_{A}\left(x_{i}\right) \vee \mu_{B}\left(x_{i}\right)} \\
& \leq \frac{1}{n} \sum_{i=1}^{n} \frac{2 \mu_{A}\left(x_{i}\right) \cdot \mu_{B}\left(x_{i}\right)}{\left(\mu_{A}\left(x_{i}\right)\right)^{2}+\left(\mu_{B}\left(x_{i}\right)\right)^{2}} \leq 1 .
\end{aligned}
$$

That is to say,

$$
\begin{aligned}
& \operatorname{IS}_{2}(A, B)=\left[\frac{1}{n} \sum_{i=1}^{n} \frac{\mu_{A}\left(x_{i}\right) \wedge \mu_{B}\left(x_{i}\right)}{\mu_{A}\left(x_{i}\right) \vee \mu_{B}\left(x_{i}\right)}, \frac{1}{n}\right. \\
& \left.\cdot \sum_{i=1}^{n} \frac{2 \mu_{A}\left(x_{i}\right) \cdot \mu_{B}\left(x_{i}\right)}{\left(\mu_{A}\left(x_{i}\right)\right)^{2}+\left(\mu_{B}\left(x_{i}\right)\right)^{2}}\right] \in D([0,1]) .
\end{aligned}
$$

The proof for $\mu_{A}\left(x_{i}\right) \geq \mu_{B}\left(x_{i}\right)$ is similar.

(SP1) If $A \in P(X)$, then $\mu_{A}(x)=0$ or $\mu_{A}(x)=1$.

$$
\begin{aligned}
\frac{1}{n} \sum_{i=1}^{n} \frac{\mu_{A}\left(x_{i}\right) \wedge \mu_{A}^{c}\left(x_{i}\right)}{\mu_{A}\left(x_{i}\right) \vee \mu_{A}^{c}\left(x_{i}\right)} & =0, \\
\frac{1}{n} \sum_{i=1}^{n} \frac{2 \mu_{A}\left(x_{i}\right) \cdot \mu_{A}^{c}\left(x_{i}\right)}{\left(\mu_{A}\left(x_{i}\right)\right)^{2}+\left(\mu_{A}^{c}\left(x_{i}\right)\right)^{2}} & =0 .
\end{aligned}
$$

Namely, $\operatorname{IS}_{2}\left(A, A^{c}\right)=[0,0]$.

(SP2) Consider

$$
\begin{gathered}
\operatorname{IS}_{2}(A, A)=\left[\frac{1}{n} \sum_{i=1}^{n} \frac{\mu_{A}\left(x_{i}\right) \wedge \mu_{A}\left(x_{i}\right)}{\mu_{A}\left(x_{i}\right) \vee \mu_{A}\left(x_{i}\right)}, \frac{1}{n}\right. \\
\left.\cdot \sum_{i=1}^{n} \frac{2 \mu_{A}\left(x_{i}\right) \cdot \mu_{A}\left(x_{i}\right)}{\left(\mu_{A}\left(x_{i}\right)\right)^{2}+\left(\mu_{A}\left(x_{i}\right)\right)^{2}}\right]=[1,1] .
\end{gathered}
$$

(SP3) It is obvious that $\operatorname{IS}_{2}(A, B)=\operatorname{IS}_{2}(B, A)$.

(SP4) If $A \subseteq B \subseteq C$, then $\mu_{A}(x) \leq \mu_{B}(x) \leq \mu_{C}(x)$. So

$$
\begin{aligned}
& \mathrm{IS}_{2}(A, C)=\left[\frac{1}{n} \sum_{i=1}^{n} \frac{\mu_{A}\left(x_{i}\right) \wedge \mu_{C}\left(x_{i}\right)}{\mu_{A}\left(x_{i}\right) \vee \mu_{C}\left(x_{i}\right)}, \frac{1}{n}\right. \\
& \left.\quad \cdot \sum_{i=1}^{n} \frac{2 \mu_{A}\left(x_{i}\right) \cdot \mu_{C}\left(x_{i}\right)}{\left(\mu_{A}\left(x_{i}\right)\right)^{2}+\left(\mu_{C}\left(x_{i}\right)\right)^{2}}\right]=\left[\frac{1}{n} \sum_{i=1}^{n} \frac{\mu_{A}\left(x_{i}\right)}{\mu_{C}\left(x_{i}\right)}, \frac{1}{n}\right. \\
& \left.\quad \cdot \sum_{i=1}^{n} \frac{2 \mu_{A}\left(x_{i}\right) \cdot \mu_{C}\left(x_{i}\right)}{\left(\mu_{A}\left(x_{i}\right)\right)^{2}+\left(\mu_{C}\left(x_{i}\right)\right)^{2}}\right]
\end{aligned}
$$

$$
\begin{aligned}
& \mathrm{IS}_{2}(A, B)=\left[\frac{1}{n} \sum_{i=1}^{n} \frac{\mu_{A}\left(x_{i}\right) \wedge \mu_{B}\left(x_{i}\right)}{\mu_{A}\left(x_{i}\right) \vee \mu_{B}\left(x_{i}\right)}, \frac{1}{n}\right. \\
& \left.\quad \cdot \sum_{i=1}^{n} \frac{2 \mu_{A}\left(x_{i}\right) \cdot \mu_{B}\left(x_{i}\right)}{\left(\mu_{A}\left(x_{i}\right)\right)^{2}+\left(\mu_{B}\left(x_{i}\right)\right)^{2}}\right]=\left[\frac{1}{n} \sum_{i=1}^{n} \frac{\mu_{A}\left(x_{i}\right)}{\mu_{B}\left(x_{i}\right)}, \frac{1}{n}\right. \\
& \left.\quad \cdot \sum_{i=1}^{n} \frac{2 \mu_{A}\left(x_{i}\right) \cdot \mu_{B}\left(x_{i}\right)}{\left(\mu_{A}\left(x_{i}\right)\right)^{2}+\left(\mu_{B}\left(x_{i}\right)\right)^{2}}\right],
\end{aligned}
$$

for the reason that

$$
\begin{aligned}
& \frac{2 \mu_{A}\left(x_{i}\right) \cdot \mu_{C}\left(x_{i}\right)}{\left(\mu_{A}\left(x_{i}\right)\right)^{2}+\left(\mu_{C}\left(x_{i}\right)\right)^{2}}-\frac{2 \mu_{A}\left(x_{i}\right) \cdot \mu_{B}\left(x_{i}\right)}{\left(\mu_{A}\left(x_{i}\right)\right)^{2}+\left(\mu_{B}\left(x_{i}\right)\right)^{2}} \\
& \quad=2 \mu_{A}\left(x_{i}\right) \\
& \quad \cdot \frac{\left(\mu_{C}\left(x_{i}\right)-\mu_{B}\left(x_{i}\right)\right)\left(\left(\mu_{A}\left(x_{i}\right)\right)^{2}-\mu_{B}\left(x_{i}\right) \mu_{C}\left(x_{i}\right)\right)}{\left[\left(\mu_{A}\left(x_{i}\right)\right)^{2}+\left(\mu_{C}\left(x_{i}\right)\right)^{2}\right]\left[\left(\mu_{A}\left(x_{i}\right)\right)^{2}+\left(\mu_{B}\left(x_{i}\right)\right)^{2}\right]} \\
& <0 .
\end{aligned}
$$

Namely,

$$
\begin{gathered}
\frac{\mu_{A}(x)}{\mu_{C}(x)} \leq \frac{\mu_{A}(x)}{\mu_{B}(x)}, \\
\frac{2 \mu_{A}\left(x_{i}\right) \cdot \mu_{C}\left(x_{i}\right)}{\left(\mu_{A}\left(x_{i}\right)\right)^{2}+\left(\mu_{C}\left(x_{i}\right)\right)^{2}} \leq \frac{2 \mu_{A}\left(x_{i}\right) \cdot \mu_{B}\left(x_{i}\right)}{\left(\mu_{A}\left(x_{i}\right)\right)^{2}+\left(\mu_{B}\left(x_{i}\right)\right)^{2}} .
\end{gathered}
$$

That is to say, $\operatorname{IS}_{2}(A, C) \leq \operatorname{IS}_{2}(A, B)$ and $\operatorname{IS}_{2}(A, C) \leq \operatorname{IS}_{2}(B, C)$. And the proofs for other cases are similar.

Definition 9. A real function ID: $F(X) \times F(X) \rightarrow D([0,1])$ is called interval distance measure on $F(X)$, if ID satisfies the following properties:

(DP1) $\operatorname{ID}\left(A, A^{c}\right)=[1,1]$ if $A$ is a crisp set.

(DP2) $\operatorname{ID}(A, A)=[0,0]$.

(DP3) $\operatorname{ID}(A, B)=\operatorname{ID}(B, A)$.

(DP4) For all $A, B, C \in F(X)$, if $A \subseteq B \subseteq C$, then

$$
\begin{aligned}
& \mathrm{ID}(A, B) \leq \mathrm{ID}(A, C), \\
& \mathrm{ID}(B, C) \leq \mathrm{ID}(A, C) .
\end{aligned}
$$

We can construct some interval distance measure formulas based on Definition 9 as follows. 
Let $X=\left\{x_{1}, x_{2}, \ldots, x_{n}\right\}$; we set

$$
\begin{aligned}
& \operatorname{ID}_{1}(A, B)=\left[\max _{i=1}^{n}\left|\mu_{A}\left(x_{i}\right)-\mu_{B}\left(x_{i}\right)\right|, 1-\frac{1}{n}\right. \\
& \left.\cdot \sum_{i=1}^{n} \frac{\mu_{A}\left(x_{i}\right) \wedge \mu_{B}\left(x_{i}\right)}{\mu_{A}\left(x_{i}\right) \vee \mu_{B}\left(x_{i}\right)}\right] \\
& \operatorname{ID}_{2}(A, B)=\left[1-\frac{1}{n} \sum_{i=1}^{n} \frac{2 \mu_{A}\left(x_{i}\right) \cdot \mu_{B}\left(x_{i}\right)}{\left(\mu_{A}\left(x_{i}\right)\right)^{2}+\left(\mu_{B}\left(x_{i}\right)\right)^{2}}, 1-\frac{1}{n}\right. \\
& \left.\cdot \sum_{i=1}^{n} \frac{\mu_{A}\left(x_{i}\right) \wedge \mu_{B}\left(x_{i}\right)}{\mu_{A}\left(x_{i}\right) \vee \mu_{B}\left(x_{i}\right)}\right] \text {; } \\
& \operatorname{ID}_{3}(A, B)=\left[\frac{1}{n} \sum_{i=1}^{n}\left|\frac{\mu_{A}\left(x_{i}\right)-\mu_{B}\left(x_{i}\right)}{\mu_{A}\left(x_{i}\right)+\mu_{B}\left(x_{i}\right)}\right|, 1-\frac{1}{n}\right. \\
& \left.\cdot \sum_{i=1}^{n} \frac{\mu_{A}\left(x_{i}\right) \wedge \mu_{B}\left(x_{i}\right)}{\mu_{A}\left(x_{i}\right) \vee \mu_{B}\left(x_{i}\right)}\right] \\
& \operatorname{ID}_{4}(A, B)=\left[1-\frac{1}{n} \sum_{i=1}^{n} \frac{2 \mu_{A}\left(x_{i}\right) \cdot \mu_{B}\left(x_{i}\right)}{\left(\mu_{A}\left(x_{i}\right)\right)^{2}+\left(\mu_{B}\left(x_{i}\right)\right)^{2}}, \frac{1}{n}\right. \\
& \left.\cdot \sum_{i=1}^{n}\left|\frac{\mu_{A}\left(x_{i}\right)-\mu_{B}\left(x_{i}\right)}{\mu_{A}\left(x_{i}\right)+\mu_{B}\left(x_{i}\right)}\right|\right] ; \\
& \operatorname{ID}_{5}(A, B)=\left[1-\frac{1}{n^{1 / p}} \sum_{i=1}^{n}\left(\frac{\mu_{A}\left(x_{i}\right) \wedge \mu_{B}\left(x_{i}\right)}{\mu_{A}\left(x_{i}\right) \vee \mu_{B}\left(x_{i}\right)}\right)^{1 / p}, 1\right. \\
& \left.-\frac{1}{n^{p}} \sum_{i=1}^{n}\left(\frac{\mu_{A}\left(x_{i}\right) \wedge \mu_{B}\left(x_{i}\right)}{\mu_{A}\left(x_{i}\right) \vee \mu_{B}\left(x_{i}\right)}\right)^{p}\right] \quad(p \geq 1) .
\end{aligned}
$$

If $\mu_{A}(x)$ and $\mu_{B}(x)$ can be integrated over the considered interval $[a, b]$, then

$$
\begin{aligned}
& \mathrm{ID}_{6}(A, B)=\left[\max _{x \in[a, b]}\left|\mu_{A}(x)-\mu_{B}(x)\right|, 1-\frac{1}{b-a}\right. \\
& \left.\cdot \int_{a}^{b} \frac{\mu_{A}(x) \wedge \mu_{B}(x)}{\mu_{A}(x) \vee \mu_{B}(x)} d x\right] ; \\
& \mathrm{ID}_{7}(A, B)=\left[1-\frac{1}{b-a}\right. \\
& \cdot \int_{a}^{b} \frac{2 \mu_{A}(x) \cdot \mu_{B}(x)}{\left(\mu_{A}(x)\right)^{2}+\left(\mu_{B}(x)\right)^{2}} d x, 1-\frac{1}{b-a} \\
& \left.\cdot \int_{a}^{b} \frac{\mu_{A}(x) \wedge \mu_{B}(x)}{\mu_{A}(x) \vee \mu_{B}(x)} d x\right] ; \\
& \mathrm{ID}_{8}(A, B)=\left[\frac{1}{b-a} \int_{a}^{b}\left|\frac{\mu_{A}(x)-\mu_{B}(x)}{\mu_{A}(x)+\mu_{B}(x)}\right| d x, 1\right. \\
& \left.-\frac{1}{b-a} \int_{a}^{b} \frac{\mu_{A}(x) \wedge \mu_{B}(x)}{\mu_{A}(x) \vee \mu_{B}(x)} d x\right] ;
\end{aligned}
$$

$$
\begin{aligned}
& \operatorname{ID}_{9}(A, B)=\left[1-\frac{1}{b-a}\right. \\
& \cdot \int_{a}^{b} \frac{2 \mu_{A}(x) \cdot \mu_{B}(x)}{\left(\mu_{A}(x)\right)^{2}+\left(\mu_{B}(x)\right)^{2}} d x, \frac{1}{b-a} \\
& \left.\cdot \int_{a}^{b}\left|\frac{\mu_{A}(x)-\mu_{B}(x)}{\mu_{A}(x)+\mu_{B}(x)}\right| d x\right] ; \\
& \mathrm{ID}_{10}(A, B)=\left[1-\frac{1}{(b-a)^{1 / p}}\right. \\
& \cdot \int_{a}^{b}\left(\frac{\mu_{A}(x) \wedge \mu_{B}(x)}{\mu_{A}(x) \vee \mu_{B}(x)}\right)^{1 / p} d x, 1-\frac{1}{(b-a)^{p}} \\
& \left.\cdot \int_{a}^{b}\left(\frac{\mu_{A}(x) \wedge \mu_{B}(x)}{\mu_{A}(x) \vee \mu_{B}(x)}\right)^{p} d x\right] \quad(p \geq 1) .
\end{aligned}
$$

The following theorem shows that the above formulas are all interval distance measures.

Theorem 10. $I D_{i}(A, B)(i=1,2, \ldots, 10)$ are interval distance measures.

Proof. When $i=3$, we have

$$
\begin{gathered}
\operatorname{ID}_{3}(A, B)=\left[\frac{1}{n} \sum_{i=1}^{n}\left|\frac{\mu_{A}\left(x_{i}\right)-\mu_{B}\left(x_{i}\right)}{\mu_{A}\left(x_{i}\right)+\mu_{B}\left(x_{i}\right)}\right|, 1\right. \\
\left.-\frac{1}{n} \sum_{i=1}^{n} \frac{\mu_{A}\left(x_{i}\right) \wedge \mu_{B}\left(x_{i}\right)}{\mu_{A}\left(x_{i}\right) \vee \mu_{B}\left(x_{i}\right)}\right] .
\end{gathered}
$$

If $\mu_{A}\left(x_{i}\right) \leq \mu_{B}\left(x_{i}\right)$, then

$$
\begin{aligned}
\left|\frac{\mu_{A}\left(x_{i}\right)-\mu_{B}\left(x_{i}\right)}{\mu_{A}\left(x_{i}\right)+\mu_{B}\left(x_{i}\right)}\right| & =\frac{\mu_{B}\left(x_{i}\right)-\mu_{A}\left(x_{i}\right)}{\mu_{A}\left(x_{i}\right)+\mu_{B}\left(x_{i}\right)}, \\
1-\frac{\mu_{A}\left(x_{i}\right) \wedge \mu_{B}\left(x_{i}\right)}{\mu_{A}\left(x_{i}\right) \vee \mu_{B}\left(x_{i}\right)} & =1-\frac{\mu_{A}\left(x_{i}\right)}{\mu_{B}\left(x_{i}\right)} \\
& =\frac{\mu_{B}\left(x_{i}\right)-\mu_{A}\left(x_{i}\right)}{\mu_{B}\left(x_{i}\right)} .
\end{aligned}
$$

Namely,

$$
\begin{aligned}
0 & \leq \frac{1}{n} \sum_{i=1}^{n}\left|\frac{\mu_{A}\left(x_{i}\right)-\mu_{B}\left(x_{i}\right)}{\mu_{A}\left(x_{i}\right)+\mu_{B}\left(x_{i}\right)}\right| \\
& \leq 1-\frac{1}{n} \sum_{i=1}^{n} \frac{\mu_{A}\left(x_{i}\right) \wedge \mu_{B}\left(x_{i}\right)}{\mu_{A}\left(x_{i}\right) \vee \mu_{B}\left(x_{i}\right)} \leq 1 .
\end{aligned}
$$

That is to say,

$$
\begin{array}{r}
\operatorname{ID}_{3}(A, B)=\left[\frac{1}{n} \sum_{i=1}^{n}\left|\frac{\mu_{A}\left(x_{i}\right)-\mu_{B}\left(x_{i}\right)}{\mu_{A}\left(x_{i}\right)+\mu_{B}\left(x_{i}\right)}\right|, 1\right. \\
\left.-\frac{1}{n} \sum_{i=1}^{n} \frac{\mu_{A}\left(x_{i}\right) \wedge \mu_{B}\left(x_{i}\right)}{\mu_{A}\left(x_{i}\right) \vee \mu_{B}\left(x_{i}\right)}\right] \in D([0,1]) .
\end{array}
$$


(DP1) If $A \in P(X)$, then $\mu_{A}(x)=0$ or $\mu_{A}(x)=1$,

$$
\begin{aligned}
& \frac{1}{n} \sum_{i=1}^{n}\left|\frac{\mu_{A}\left(x_{i}\right)-\mu_{A}^{c}\left(x_{i}\right)}{\mu_{A}\left(x_{i}\right)+\mu_{A}^{c}\left(x_{i}\right)}\right|=1, \\
& 1-\frac{1}{n} \sum_{i=1}^{n} \frac{\mu_{A}\left(x_{i}\right) \wedge \mu_{A}^{c}\left(x_{i}\right)}{\mu_{A}\left(x_{i}\right) \vee \mu_{A}^{c}\left(x_{i}\right)}=1 .
\end{aligned}
$$

Namely, $\mathrm{ID}_{3}\left(A, A^{c}\right)=[1,1]$

(DP2) Consider

$$
\begin{gathered}
\operatorname{ID}_{3}(A, A)=\left[\frac{1}{n} \sum_{i=1}^{n}\left|\frac{\mu_{A}\left(x_{i}\right)-\mu_{A}\left(x_{i}\right)}{\mu_{A}\left(x_{i}\right)+\mu_{A}\left(x_{i}\right)}\right|, 1\right. \\
\left.-\frac{1}{n} \sum_{i=1}^{n} \frac{\mu_{A}\left(x_{i}\right) \wedge \mu_{A}\left(x_{i}\right)}{\mu_{A}\left(x_{i}\right) \vee \mu_{A}\left(x_{i}\right)}\right]=[0,0] .
\end{gathered}
$$

(DP3) It is obvious that $\operatorname{ID}_{3}(A, B)=\operatorname{ID}_{3}(B, A)$.

(DP4) If $A \subseteq B \subseteq C$, then $\mu_{A}(x) \leq \mu_{B}(x) \leq \mu_{C}(x)$ and

$$
\begin{aligned}
\mathrm{ID}_{3}(A, B)=\left[\frac{1}{n} \sum_{i=1}^{n}\left|\frac{\mu_{A}\left(x_{i}\right)-\mu_{B}\left(x_{i}\right)}{\mu_{A}\left(x_{i}\right)+\mu_{B}\left(x_{i}\right)}\right|, 1\right. \\
\left.-\frac{1}{n} \sum_{i=1}^{n} \frac{\mu_{A}\left(x_{i}\right) \wedge \mu_{B}\left(x_{i}\right)}{\mu_{A}\left(x_{i}\right) \vee \mu_{B}\left(x_{i}\right)}\right] \\
=\left[\frac{1}{n} \sum_{i=1}^{n} \frac{\mu_{B}\left(x_{i}\right)-\mu_{A}\left(x_{i}\right)}{\mu_{A}\left(x_{i}\right)+\mu_{B}\left(x_{i}\right)}, \frac{1}{n} \sum_{i=1}^{n} 1-\frac{\mu_{A}\left(x_{i}\right)}{\mu_{B}\left(x_{i}\right)}\right], \\
\mathrm{ID}_{3}(A, C)=\left[\frac{1}{n} \sum_{i=1}^{n}\left|\frac{\mu_{A}\left(x_{i}\right)-\mu_{C}\left(x_{i}\right)}{\mu_{A}\left(x_{i}\right)+\mu_{C}\left(x_{i}\right)}\right|, 1\right. \\
\left.-\frac{1}{n} \sum_{i=1}^{n} \frac{\mu_{A}\left(x_{i}\right) \wedge \mu_{C}\left(x_{i}\right)}{\mu_{A}\left(x_{i}\right) \vee \mu_{C}\left(x_{i}\right)}\right] \\
=\left[\frac{1}{n} \sum_{i=1}^{n} \frac{\mu_{C}\left(x_{i}\right)-\mu_{A}\left(x_{i}\right)}{\mu_{A}\left(x_{i}\right)+\mu_{C}\left(x_{i}\right)}, \frac{1}{n} \sum_{i=1}^{n} 1-\frac{\mu_{A}\left(x_{i}\right)}{\mu_{C}\left(x_{i}\right)}\right],
\end{aligned}
$$

for the reason that

$$
\begin{aligned}
& \frac{\mu_{B}\left(x_{i}\right)-\mu_{A}\left(x_{i}\right)}{\mu_{A}\left(x_{i}\right)+\mu_{B}\left(x_{i}\right)}=1-\frac{2 \mu_{A}\left(x_{i}\right)}{\mu_{A}\left(x_{i}\right)+\mu_{B}\left(x_{i}\right)}, \\
& \frac{\mu_{C}\left(x_{i}\right)-\mu_{A}\left(x_{i}\right)}{\mu_{A}\left(x_{i}\right)+\mu_{C}\left(x_{i}\right)}=1-\frac{2 \mu_{A}\left(x_{i}\right)}{\mu_{A}\left(x_{i}\right)+\mu_{C}\left(x_{i}\right)} .
\end{aligned}
$$

We have

$$
\begin{gathered}
\frac{\mu_{B}\left(x_{i}\right)-\mu_{A}\left(x_{i}\right)}{\mu_{A}\left(x_{i}\right)+\mu_{B}\left(x_{i}\right)} \leq \frac{\mu_{C}\left(x_{i}\right)-\mu_{A}\left(x_{i}\right)}{\mu_{A}\left(x_{i}\right)+\mu_{C}\left(x_{i}\right)}, \\
1-\frac{\mu_{A}\left(x_{i}\right) \wedge \mu_{B}\left(x_{i}\right)}{\mu_{A}\left(x_{i}\right) \vee \mu_{B}\left(x_{i}\right)} \leq 1-\frac{\mu_{A}\left(x_{i}\right) \wedge \mu_{C}\left(x_{i}\right)}{\mu_{A}\left(x_{i}\right) \vee \mu_{C}\left(x_{i}\right)} .
\end{gathered}
$$

Namely, $\mathrm{ID}_{3}(A, B) \leq \mathrm{ID}_{3}(A, C), \mathrm{ID}_{3}(B, C) \leq \operatorname{ID}_{3}(A, C)$.

The proofs for other cases are similar.
Definition 11. A real function II: $F(X) \times F(X) \rightarrow D([0,1])$ is called interval inclusion measure on $F(X)$, if II satisfies the following properties:

(IP1) $\operatorname{II}(X, \Phi)=[0,0]$.

(IP2) $\operatorname{II}(A, B)=[1,1] \Leftrightarrow A \subseteq B$.

(IP3) For all $A, B, C \in F(X)$, if $A \subseteq B \subseteq C$, then

$$
\begin{aligned}
& \mathrm{II}(C, A) \leq \mathrm{II}(B, A), \\
& \mathrm{II}(C, A) \leq \mathrm{II}(C, B) .
\end{aligned}
$$

We can construct some interval inclusion measure formulas based on Definition 11 as follows.

Let $X=\left\{x_{1}, x_{2}, \ldots, x_{n}\right\}$,

$$
\begin{array}{r}
\mathrm{II}_{1}(A, B)=\left[\frac{1}{n} \sum_{i=1}^{n}\left(\frac{\mu_{A}\left(x_{i}\right) \wedge \mu_{B}\left(x_{i}\right)}{\mu_{A}\left(x_{i}\right)}\right)^{p}, \frac{1}{n}\right. \\
\left.\cdot \sum_{i=1}^{n}\left(\frac{\mu_{A}\left(x_{i}\right) \wedge \mu_{B}\left(x_{i}\right)}{\mu_{A}\left(x_{i}\right)}\right)^{1 / p}\right] \quad(p \geq 1) ; \\
\mathrm{II}_{2}(A, B)=\left[\frac{1}{n} \sum_{i=1}^{n}\left(\frac{\mu_{B}\left(x_{i}\right)}{\mu_{A}\left(x_{i}\right) \vee \mu_{B}\left(x_{i}\right)}\right)^{p}, \frac{1}{n}\right. \\
\left.\cdot \sum_{i=1}^{n}\left(\frac{\mu_{B}\left(x_{i}\right)}{\mu_{A}\left(x_{i}\right) \vee \mu_{B}\left(x_{i}\right)}\right)^{1 / p}\right] \quad(p \geq 1) .
\end{array}
$$

If $\mu_{A}(x)$ and $\mu_{B}(x)$ can be integrated over the considered interval $[a, b]$, we set

$$
\begin{aligned}
& \mathrm{II}_{3}(A, B)=\left[\frac{1}{(b-a)}\right. \\
& \cdot \int_{a}^{b}\left(\frac{\mu_{A}(x) \wedge \mu_{B}(x)}{\mu_{A}(x)}\right)^{p} d x, \frac{1}{(b-a)} \\
& \left.\cdot \int_{a}^{b}\left(\frac{\mu_{A}(x) \wedge \mu_{B}(x)}{\mu_{A}(x)}\right)^{1 / p} d x\right] \quad(p \geq 1) ; \\
& \mathrm{II}_{4}(A, B)=\left[\frac{1}{(b-a)} \int_{a}^{b}\left(\frac{\mu_{B}(x)}{\mu_{A}(x) \vee \mu_{B}(x)}\right)^{p} d x, \frac{1}{(b-a)}\right. \\
& \left.\cdot \int_{a}^{b}\left(\frac{\mu_{B}(x)}{\mu_{A}(x) \vee \mu_{B}(x)}\right)^{1 / p} d x\right] \quad(p \geq 1) .
\end{aligned}
$$

The following theorem shows that the above formulas are all interval inclusion measures.

Theorem 12. $I I_{i}(A, B)(i=1,2,3,4)$ are interval inclusion measures. 
Proof. When $i=1$, we have

$$
\begin{array}{r}
\mathrm{II}_{1}(A, B)=\left[\frac{1}{n} \sum_{i=1}^{n}\left(\frac{\mu_{A}\left(x_{i}\right) \wedge \mu_{B}\left(x_{i}\right)}{\mu_{A}\left(x_{i}\right)}\right)^{p}, \frac{1}{n}\right. \\
\left.\cdot \sum_{i=1}^{n}\left(\frac{\mu_{A}\left(x_{i}\right) \wedge \mu_{B}\left(x_{i}\right)}{\mu_{A}\left(x_{i}\right)}\right)^{1 / p}\right] \quad(p \geq 1) .
\end{array}
$$

It is obvious that

$$
\begin{aligned}
0 & \leq \frac{1}{n} \sum_{i=1}^{n}\left(\frac{\mu_{A}\left(x_{i}\right) \wedge \mu_{B}\left(x_{i}\right)}{\mu_{A}\left(x_{i}\right)}\right)^{p} \\
& \leq \frac{1}{n} \sum_{i=1}^{n}\left(\frac{\mu_{A}\left(x_{i}\right) \wedge \mu_{B}\left(x_{i}\right)}{\mu_{A}\left(x_{i}\right)}\right)^{1 / p} \leq 1 .
\end{aligned}
$$

(IP1) Consider

$$
\begin{gathered}
\mathrm{II}_{1}(X, \phi)=\left[\frac{1}{n} \sum_{i=1}^{n}\left(\frac{\mu_{X}\left(x_{i}\right) \wedge \mu_{\phi}\left(x_{i}\right)}{\mu_{X}\left(x_{i}\right)}\right)^{p}, \frac{1}{n}\right. \\
\left.\cdot \sum_{i=1}^{n}\left(\frac{\mu_{X}\left(x_{i}\right) \wedge \mu_{\phi}\left(x_{i}\right)}{\mu_{X}\left(x_{i}\right)}\right)^{1 / p}\right]=[0,0] .
\end{gathered}
$$

(IP2) Consider

$$
\begin{aligned}
& \mathrm{II}_{1}(A, B)=[1,1] \\
& \Longleftrightarrow\left[\frac{1}{n} \sum_{i=1}^{n}\left(\frac{\mu_{A}\left(x_{i}\right) \wedge \mu_{B}\left(x_{i}\right)}{\mu_{A}\left(x_{i}\right)}\right)^{p}, \frac{1}{n}\right. \\
& \left.\cdot \sum_{i=1}^{n}\left(\frac{\mu_{A}\left(x_{i}\right) \wedge \mu_{B}\left(x_{i}\right)}{\mu_{A}\left(x_{i}\right)}\right)^{1 / p}\right]=[1,1] \\
& \Longleftrightarrow \frac{\mu_{A}\left(x_{i}\right) \wedge \mu_{B}\left(x_{i}\right)}{\mu_{A}\left(x_{i}\right)}=1 \\
& \Longleftrightarrow A \subseteq B .
\end{aligned}
$$

(IP3) If $A \subseteq B \subseteq C$, then $\mu_{A}(x) \leq \mu_{B}(x) \leq \mu_{C}(x)$,

$$
\begin{gathered}
\mathrm{II}_{1}(C, A)=\left[\frac{1}{n} \sum_{i=1}^{n}\left(\frac{\mu_{C}\left(x_{i}\right) \wedge \mu_{A}\left(x_{i}\right)}{\mu_{C}\left(x_{i}\right)}\right)^{p}, \frac{1}{n}\right. \\
\left.\cdot \sum_{i=1}^{n}\left(\frac{\mu_{C}\left(x_{i}\right) \wedge \mu_{A}\left(x_{i}\right)}{\mu_{C}\left(x_{i}\right)}\right)^{1 / p}\right]=\left[\frac{1}{n}\right. \\
\left.\cdot \sum_{i=1}^{n}\left(\frac{\mu_{A}\left(x_{i}\right)}{\mu_{C}\left(x_{i}\right)}\right)^{p}, \frac{1}{n} \sum_{i=1}^{n}\left(\frac{\mu_{A}\left(x_{i}\right)}{\mu_{C}\left(x_{i}\right)}\right)^{1 / p}\right],
\end{gathered}
$$

$$
\begin{gathered}
\mathrm{II}_{1}(B, A)=\left[\frac{1}{n} \sum_{i=1}^{n}\left(\frac{\mu_{B}\left(x_{i}\right) \wedge \mu_{A}\left(x_{i}\right)}{\mu_{B}\left(x_{i}\right)}\right)^{p}, \frac{1}{n}\right. \\
\left.\cdot \sum_{i=1}^{n}\left(\frac{\mu_{B}\left(x_{i}\right) \wedge \mu_{A}\left(x_{i}\right)}{\mu_{B}\left(x_{i}\right)}\right)^{1 / p}\right]=\left[\frac{1}{n}\right. \\
\left.\cdot \sum_{i=1}^{n}\left(\frac{\mu_{A}\left(x_{i}\right)}{\mu_{B}\left(x_{i}\right)}\right)^{p}, \frac{1}{n} \sum_{i=1}^{n}\left(\frac{\mu_{A}\left(x_{i}\right)}{\mu_{B}\left(x_{i}\right)}\right)^{1 / p}\right] .
\end{gathered}
$$

So

$$
\begin{gathered}
\frac{1}{n} \sum_{i=1}^{n}\left(\frac{\mu_{A}\left(x_{i}\right)}{\mu_{C}\left(x_{i}\right)}\right)^{p} \leq \frac{1}{n} \sum_{i=1}^{n}\left(\frac{\mu_{A}\left(x_{i}\right)}{\mu_{B}\left(x_{i}\right)}\right)^{p}, \\
\frac{1}{n} \sum_{i=1}^{n}\left(\frac{\mu_{A}\left(x_{i}\right)}{\mu_{C}\left(x_{i}\right)}\right)^{1 / p} \leq \frac{1}{n} \sum_{i=1}^{n}\left(\frac{\mu_{A}\left(x_{i}\right)}{\mu_{B}\left(x_{i}\right)}\right)^{1 / p} .
\end{gathered}
$$

Namely, $\mathrm{II}_{1}(C, A) \leq \mathrm{II}_{1}(B, A)$ and $\mathrm{II}_{1}(C, A) \leq \mathrm{II}_{1}(C, B)$. The proofs for other cases are similar.

4.2. The Relationships with the above Definitions. In this section, some conclusions about the relationships with those definitions are presented. From the follow theorems and corollaries, any two can be transformed into each other.

Theorem 13. If IE is interval entropy of fuzzy sets, $\forall A, B \in$ $F(X), \forall x \in X$,

$$
\begin{aligned}
& f_{1}(A, B)(x)=\frac{\mu_{A}(x) \wedge \mu_{B}(x)}{2\left(\mu_{A}(x) \vee \mu_{B}(x)\right)}, \\
& f_{2}(A, B)(x)=\frac{1-\left|\mu_{A}(x)-\mu_{B}(x)\right|}{2}, \\
& f_{3}(A, B)(x)=\frac{1+\left|\mu_{A}(x)-\mu_{B}(x)\right|}{2},
\end{aligned}
$$

then

(1) $\operatorname{IE}\left(f_{i}(A, B)\right)(i=1,2,3)$ are interval similarity measures of fuzzy sets,

(2) $I E^{c}\left(f_{i}(A, B)\right)(i=1,2,3)$ are interval distance measures of fuzzy sets,

(3) $\operatorname{IE}\left(f_{i}(B, A \cup B)\right)(i=1,2,3)$ are interval inclusion measures of the fuzzy sets.

Proof. (1) When $i=3$, we have the following:

(SP1) If $A \in P(X)$, then $\forall x \in X$,

$$
f_{3}\left(A, A^{c}\right)(x)=\frac{1+\left|\mu_{A}(x)-\mu_{A}^{c}(x)\right|}{2}=1 .
$$

Namely, $f_{3}\left(A, A^{c}\right) \in P(X)$, so $\operatorname{IE}\left(f_{3}\left(A, A^{c}\right)\right)=[0,0]$.

(SP2) $\forall x \in X$, we have

$$
f_{3}(A, A)(x)=\frac{1+\left|\mu_{A}(x)-\mu_{A}(x)\right|}{2}=\frac{1}{2} .
$$

So $\operatorname{IE}\left(f_{3}\left(A, A^{c}\right)\right)=[1,1]$.

(SP3) It is obvious that $\operatorname{IE}\left(f_{3}(A, B)\right)=\operatorname{IE}\left(f_{3}(B, A)\right)$. 
(SP4) If $A \subseteq B \subseteq C$, then $\mu_{A}(x) \leq \mu_{B}(x) \leq \mu_{C}(x)$, so

$$
\begin{aligned}
& \frac{1}{2} \leq \frac{1+\left|\mu_{A}(x)-\mu_{B}(x)\right|}{2} \leq \frac{1+\left|\mu_{A}(x)-\mu_{C}(x)\right|}{2}, \\
& \frac{1}{2} \leq \frac{1+\left|\mu_{B}(x)-\mu_{C}(x)\right|}{2} \leq \frac{1+\left|\mu_{A}(x)-\mu_{C}(x)\right|}{2} .
\end{aligned}
$$

Namely,

$$
\begin{aligned}
& \operatorname{IE}\left(f_{3}(A, B)\right) \geq \operatorname{IE}\left(f_{3}(A, C)\right), \\
& \operatorname{IE}\left(f_{3}(B, C)\right) \geq \operatorname{IE}\left(f_{3}(A, C)\right) .
\end{aligned}
$$

The proofs for other cases are similar.

(2) When $i=1$, let $\operatorname{ID}(A, B)=\operatorname{IE}^{c}\left(f_{1}(A, B)\right)$.

(DP1) $\forall A \in P(X)$, we have $f_{1}(A, A)(x)=0$, so $f_{1}(A, A) \in$ $P(X)$ and

$$
\operatorname{IE}^{c}\left(f_{1}(A, A)\right)=[1,1] .
$$

(DP2) Let $\operatorname{ID}(A, A)=\operatorname{IE}^{c}(f 1(A, A))$, for the reason that $f_{1}(A, A)(x)=1 / 2$, so

$$
\operatorname{IE}^{c}\left(f_{1}(A, A)\right)=[0,0] .
$$

(DP3) It is obvious that $\operatorname{ID}(A, B)=\operatorname{ID}(B, A)$.

(DP4) If $A \subseteq B \subseteq C$, then $\mu_{A}(x) \leq \mu_{B}(x) \leq \mu_{C}(x)$. For the reason that

$$
\begin{gathered}
\frac{\mu_{A}(x) \wedge \mu_{B}(x)}{2\left(\mu_{A}(x) \vee \mu_{B}(x)\right)}=\frac{\mu_{A}(x)}{2 \mu_{B}(x)}, \\
\frac{\mu_{A}(x) \wedge \mu_{C}(x)}{2\left(\mu_{A}(x) \vee \mu_{C}(x)\right)}=\frac{\mu_{A}(x)}{2 \mu_{C}(x)}, \\
\frac{\mu_{B}(x) \wedge \mu_{C}(x)}{2\left(\mu_{B}(x) \vee \mu_{C}(x)\right)}=\frac{\mu_{B}(x)}{2 \mu_{C}(x)},
\end{gathered}
$$

we have

$$
\begin{aligned}
& \frac{\mu_{A}(x)}{2 \mu_{C}(x)} \leq \frac{\mu_{A}(x)}{2 \mu_{B}(x)} \leq \frac{1}{2}, \\
& \frac{\mu_{A}(x)}{2 \mu_{C}(x)} \leq \frac{\mu_{B}(x)}{2 \mu_{C}(x)} \leq \frac{1}{2} .
\end{aligned}
$$

Namely,

$$
\begin{aligned}
& \operatorname{IE}^{c}\left(f_{1}(A, B)\right) \leq \operatorname{IE}^{c}\left(f_{1}(A, C)\right), \\
& \operatorname{IE}^{c}\left(f_{1}(B, C)\right) \leq \operatorname{IE}^{c}\left(f_{1}(A, C)\right) .
\end{aligned}
$$

The proofs for other cases are similar.

(3) When $i=1$, we set $\operatorname{II}(A, B)=\operatorname{IE}\left(f_{1}(B, A \cup B)\right)$.

$(\mathrm{IP} 1) \operatorname{II}(X, \Phi)=\operatorname{IE}\left(f_{1}(\Phi, X \cup \Phi)\right)=\operatorname{IE}\left(f_{1}(\Phi, X)\right), \forall x \in$ $X, f_{1}(\Phi, X)=0$, so $f_{1}(\Phi, X) \in P(X)$ and $\operatorname{II}(X, \Phi)=$ $\operatorname{IE}\left(f_{1}(\Phi, X)\right)=[0,0]$.
(IP2) Consider

$$
\begin{aligned}
\operatorname{II}(A, B) & =[1,1] \\
& \Longleftrightarrow \operatorname{IE}\left(f_{1}(B, A \cup B)\right)=[1,1] \\
& \Longleftrightarrow f_{1}(B, A \cup B)(x) \equiv \frac{1}{2}, \quad \forall x \in X \\
& \Longleftrightarrow \frac{\mu_{B}(x) \wedge\left(\mu_{A}(x) \vee \mu_{B}(x)\right)}{2\left(\mu_{A}(x) \vee \mu_{B}(x)\right)} \equiv \frac{1}{2}, \quad \forall x \in X \\
& \Longleftrightarrow \mu_{A}(x) \vee \mu_{B}(x)=\mu_{B}(x), \quad \forall x \in X \\
& \Longleftrightarrow \mu_{A}(x) \leq \mu_{B}(x), \quad \forall x \in X \\
& \Longleftrightarrow A \subseteq B .
\end{aligned}
$$

(IP3) If $A \subseteq B \subseteq C$, then $\mu_{A}(x) \leq \mu_{B}(x) \leq \mu_{C}(x)$ and

$$
\begin{aligned}
& \operatorname{II}(C, A)=\operatorname{IE}\left(f_{1}(A, C \cup A)\right)=\operatorname{IE}\left(f_{1}(A, C)\right), \\
& \operatorname{II}(B, A)=\operatorname{IE}\left(f_{1}(A, B \cup A)\right)=\operatorname{IE}\left(f_{1}(A, B)\right), \\
& \operatorname{II}(C, B)=\operatorname{IE}\left(f_{1}(B, C \cup B)\right)=\operatorname{IE}\left(f_{1}(B, C)\right) .
\end{aligned}
$$

Owing to the fact that

$$
\begin{aligned}
& \frac{\mu_{A}(x) \wedge \mu_{B}(x)}{2\left(\mu_{A}(x) \vee \mu_{B}(x)\right)}=\frac{\mu_{A}(x)}{2 \mu_{B}(x)}, \\
& \frac{\mu_{A}(x) \wedge \mu_{C}(x)}{2\left(\mu_{A}(x) \vee \mu_{C}(x)\right)}=\frac{\mu_{A}(x)}{2 \mu_{C}(x)}, \\
& \frac{\mu_{B}(x) \wedge \mu_{C}(x)}{2\left(\mu_{B}(x) \vee \mu_{C}(x)\right)}=\frac{\mu_{B}(x)}{2 \mu_{C}(x)},
\end{aligned}
$$

then $\forall x \in X, \mu_{A}(x) / 2 \mu_{C}(x) \leq \mu_{A}(x) / 2 \mu_{B}(x) \leq 1 / 2, \mu_{A}(x) /$ $2 \mu_{C}(x) \leq \mu_{B}(x) / 2 \mu_{C}(x) \leq 1 / 2$.

Namely, $\mathrm{II}(C, A) \leq \mathrm{II}(B, A), \mathrm{II}(C, A) \leq \mathrm{II}(C, B)$.

The proofs for other cases are similar.

Corollary 14. $\operatorname{IE}\left(f_{i}(A \cap B, A)\right)(i=1,2,3)$ are interval inclusion measures of the fuzzy sets.

Proof. When $i=1$, we set $\operatorname{II}(A, B)=\operatorname{IE}\left(f_{1}(A \cap B, A)\right)$.

(IP1) $\operatorname{II}(X, \Phi)=\operatorname{IE}\left(f_{1}(X \cap \Phi, X)\right)=\operatorname{IE}\left(f_{1}(\Phi, X)\right)$, $\forall x \in X, f_{1}(\Phi, X)=0$, so $f_{1}(\Phi, X) \in P(X)$ and $\operatorname{II}(X, \Phi)=$ $\operatorname{IE}\left(f_{1}(\Phi, X)\right)=[0,0]$.

(IP2) Consider

$$
\begin{aligned}
\operatorname{II}(A, B) & =[1,1] \\
& \Longleftrightarrow \operatorname{IE}\left(f_{1}(A \cap B, A)\right)=[1,1] \\
& \Longleftrightarrow f_{1}(A \cap B, A)(x) \equiv \frac{1}{2}, \quad \forall x \in X \\
& \Longleftrightarrow \frac{\mu_{A}(x) \wedge \mu_{B}(x)}{2 \mu_{A}(x)} \equiv \frac{1}{2}, \quad \forall x \in X
\end{aligned}
$$




$$
\begin{aligned}
& \Longleftrightarrow \mu_{A}(x) \wedge \mu_{B}(x)=\mu_{A}(x), \quad \forall x \in X \\
& \Longleftrightarrow \mu_{A}(x) \leq \mu_{B}(x), \quad \forall x \in X \\
& \Longleftrightarrow A \subseteq B .
\end{aligned}
$$

(IP3) If $A \subseteq B \subseteq C$, then $\mu_{A}(x) \leq \mu_{B}(x) \leq \mu_{C}(x)$ and

$$
\begin{aligned}
& \operatorname{II}(C, A)=\operatorname{IE}\left(f_{1}(C \cap A, C)\right)=\operatorname{IE}\left(f_{1}(A, C)\right) ; \\
& \operatorname{II}(B, A)=\operatorname{IE}\left(f_{1}(B \cap A, B)\right)=\operatorname{IE}\left(f_{1}(A, B)\right) ; \\
& \operatorname{II~}(C, B)=\operatorname{IE}\left(f_{1}(C \cap B, C)\right)=\operatorname{IE}\left(f_{1}(B, C)\right) .
\end{aligned}
$$

In view of the fact that

$$
\begin{aligned}
& \frac{\mu_{A}(x) \wedge \mu_{B}(x)}{2\left(\mu_{A}(x) \vee \mu_{B}(x)\right)}=\frac{\mu_{A}(x)}{2 \mu_{B}(x)}, \\
& \frac{\mu_{A}(x) \wedge \mu_{C}(x)}{2\left(\mu_{A}(x) \vee \mu_{C}(x)\right)}=\frac{\mu_{A}(x)}{2 \mu_{C}(x)}, \\
& \frac{\mu_{B}(x) \wedge \mu_{C}(x)}{2\left(\mu_{B}(x) \vee \mu_{C}(x)\right)}=\frac{\mu_{B}(x)}{2 \mu_{C}(x)},
\end{aligned}
$$

then we have $\forall x \in X, \mu_{A}(x) / 2 \mu_{C}(x) \leq \mu_{A}(x) / 2 \mu_{B}(x) \leq 1 / 2$, $\mu_{A}(x) / 2 \mu_{C}(x) \leq \mu_{B}(x) / 2 \mu_{C}(x) \leq 1 / 2$.

Namely, $\mathrm{II}(C, A) \leq \mathrm{II}(B, A), \mathrm{II}(C, A) \leq \mathrm{II}(C, B)$.

The proofs for other cases are similar.

For example, if

$$
\begin{gathered}
\operatorname{IE}(A)=\left[\frac{1}{n} \sum_{i=1}^{n} 4\left(\left(\mu_{A}\left(x_{i}\right)\right)^{2} \wedge\left(\mu_{A}^{c}\left(x_{i}\right)\right)^{2}\right), \frac{1}{n}\right. \\
\left.\cdot \sum_{i=1}^{n} \frac{\mu_{A}\left(x_{i}\right) \wedge \mu_{A}^{c}\left(x_{i}\right)}{\mu_{A}\left(x_{i}\right) \vee \mu_{A}^{c}\left(x_{i}\right)}\right],
\end{gathered}
$$

then

$$
\begin{gathered}
\operatorname{IS}_{11}(A, B)=\operatorname{IE}\left(f_{3}(A, B)\right)=\left[\frac{1}{n}\right. \\
\cdot \sum_{i=1}^{n}\left(1-\left|\mu_{A}\left(x_{i}\right)-\mu_{B}\left(x_{i}\right)\right|\right)^{2}, \frac{1}{n} \\
\left.\cdot \sum_{i=1}^{n} \frac{1-\left|\mu_{A}\left(x_{i}\right)-\mu_{B}\left(x_{i}\right)\right|}{1+\left|\mu_{A}\left(x_{i}\right)-\mu_{B}\left(x_{i}\right)\right|}\right]
\end{gathered}
$$

is an interval similarity measure. And

$$
\begin{aligned}
\operatorname{ID}_{11}(A, B)=\operatorname{IE}^{c}\left(f_{3}(A, B)\right) & \\
= & {\left[\frac{1}{n} \sum_{i=1}^{n} \frac{2\left|\mu_{A}\left(x_{i}\right)-\mu_{B}\left(x_{i}\right)\right|}{1+\left|\mu_{A}\left(x_{i}\right)-\mu_{B}\left(x_{i}\right)\right|}, 1\right.} \\
- & \left.\frac{1}{n} \sum_{i=1}^{n}\left(1-\left|\mu_{A}\left(x_{i}\right)-\mu_{B}\left(x_{i}\right)\right|\right)^{2}\right]
\end{aligned}
$$

is an interval distance measure. And

$$
\begin{gathered}
\mathrm{II}_{5}(A, B)=\operatorname{IE}\left(f_{3}(B, A \cup B)\right)=\left[\frac{1}{n}\right. \\
\cdot \sum_{i=1}^{n}\left(1-\left|\mu_{B}\left(x_{i}\right)-\mu_{A}\left(x_{i}\right) \vee \mu_{B}\left(x_{i}\right)\right|\right)^{2}, \frac{1}{n} \\
\left.\cdot \sum_{i=1}^{n} \frac{1-\left|\mu_{B}\left(x_{i}\right)-\mu_{A}\left(x_{i}\right) \vee \mu_{B}\left(x_{i}\right)\right|}{1+\left|\mu_{B}\left(x_{i}\right)-\mu_{A}\left(x_{i}\right) \vee \mu_{B}\left(x_{i}\right)\right|}\right], \\
\mathrm{II}_{6}(A, B)=\operatorname{IE}\left(f_{3}(A \cap B, A)\right)=\left[\frac{1}{n}\right. \\
\cdot \sum_{i=1}^{n}\left(1-\left|\mu_{A}\left(x_{i}\right) \wedge \mu_{B}\left(x_{i}\right)-\mu_{A}\left(x_{i}\right)\right|\right)^{2}, \frac{1}{n} \\
\left.\cdot \sum_{i=1}^{n} \frac{1-\left|\mu_{A}\left(x_{i}\right) \wedge \mu_{B}\left(x_{i}\right)-\mu_{A}\left(x_{i}\right)\right|}{1+\left|\mu_{A}\left(x_{i}\right) \wedge \mu_{B}\left(x_{i}\right)-\mu_{A}\left(x_{i}\right)\right|}\right]
\end{gathered}
$$

are interval inclusion measures.

Theorem 15. For $A, B \in F(X)$, IS is interval similarity measure of fuzzy sets; then

(1) IS $\left(A, A^{c}\right)$ is interval entropy of the fuzzy sets;

(2) $I S^{C}(A, B)$ is interval distance measure;

(3) $I S(B, A \cup B)$ is interval inclusion measure.

Proof. The proof is as follows:

(1)

(EP1) If $A \in P(X)$, then IS $\left(A, A^{c}\right)=[0,0]$.

(EP2) If $\forall x \in X, \mu_{A}(x) \equiv 1 / 2$, then $A(X)=A^{c}(X)$ and $\operatorname{IS}\left(A, A^{c}\right)=\operatorname{IS}(A, A)=[1,1]$.

(EP3) When $\forall x \in X, \mu_{A_{2}}(x) \leq \mu_{A_{1}}(x) \leq 1 / 2$, we have $\mu_{A_{2}}(x) \leq \mu_{A_{1}}(x) \leq 1 / 2 \leq \mu_{A_{1}}^{c}(x) \leq \mu_{A_{2}}^{c}(x)$, so

$$
A_{2} \subseteq A_{1} \subseteq A_{1}^{c} \subseteq A_{2}^{c} .
$$

(EP4) It is obvious that $\operatorname{IS}\left(A, A^{c}\right)=\operatorname{IS}\left(A^{c}, A\right)$.

Namely,

$$
\operatorname{IS}\left(A_{2}, A_{2}^{c}\right) \leq \operatorname{IS}\left(A_{1}, A_{2}^{c}\right) \leq \operatorname{IS}\left(A_{1}, A_{1}^{c}\right) .
$$

When $\forall x \in X, \mu_{A_{2}}(x) \geq \mu_{A_{1}}(x) \geq 1 / 2$, the proof is similar.

(2) Let $\operatorname{ID}(A, B)=\operatorname{IS}^{\mathcal{C}}(A, B)$.

(DP1) If $A \in P(X)$, then $\operatorname{IS}^{c}\left(A, A^{c}\right)=[0,0]^{c}=[1,1]$.

(DP2) $\operatorname{IS}^{\mathcal{C}}(A, A)=[1,1]^{c}=[0,0]$.

(DP3) It is obvious that $\operatorname{IS}^{c}(A, B)=\operatorname{IS}^{c}(B, A)$.

(DP4) If $A \subseteq B \subseteq C$, then $\operatorname{IS}(A, C) \leq \operatorname{IS}(A, B)$, $\operatorname{IS}(A, C) \leq \operatorname{IS}(B, C)$. Namely,

$$
\begin{aligned}
& \operatorname{IS}^{\mathcal{c}}(A, B) \leq \operatorname{IS}^{\mathcal{c}}(A, C), \\
& \operatorname{IS}^{\mathcal{c}}(B, C) \leq \operatorname{IS}^{\mathcal{c}}(A, C) .
\end{aligned}
$$


(3) Let $\operatorname{II}(A, B)=\operatorname{IS}(B, A \cup B)$.

(IP1) $\operatorname{II}(X, \Phi)=\operatorname{IS}(\Phi, X \cup \Phi)=\operatorname{IS}(\Phi, X)$, for $\Phi, X \epsilon$ $P(X)$, so $\operatorname{II}(X, \Phi)=\operatorname{IS}(\Phi, X)=[0,0]$.

(IP2) $\operatorname{II}(A, B)=[1,1] \Leftrightarrow \operatorname{IS}(B, A \cup B)=[1,1] \Leftrightarrow$ $A \cup B=B \Leftrightarrow A \subseteq B$.

(IP3) If $A \subseteq B \subseteq C$, then $\operatorname{IS}(A, C) \leq \operatorname{IS}(A, B)$, $\operatorname{IS}(A, C) \leq \operatorname{IS}(B, C)$ and

$$
\begin{aligned}
& \mathrm{II}(C, A)=\operatorname{IS}(A, A \cup C)=\operatorname{IS}(A, C) ; \\
& \mathrm{II}(B, A)=\operatorname{IS}(A, A \cup B)=\operatorname{IS}(A, B) ; \\
& \mathrm{II}(C, B)=\operatorname{IS}(B, B \cup C)=\operatorname{IS}(B, C) .
\end{aligned}
$$

Namely, $\mathrm{II}(C, A) \leq \mathrm{II}(B, A), \mathrm{II}(C, A) \leq \mathrm{II}(C, B)$.

Corollary 16. IS $(A \cap B, B)$ is interval inclusion measure.

Proof. Let II $(A, B)=\operatorname{IS}(A \cap B, B)$.

(IP1) $\operatorname{II}(X, \Phi)=\operatorname{IS}(X \cap \Phi, X)=\operatorname{IS}(\Phi, X)$. For $\Phi, X \in$ $P(X)$, we have $\operatorname{II}(X, \Phi)=\operatorname{IS}(\Phi, X)=[0,0]$.

(IP2) $\operatorname{II}(A, B)=[1,1] \Leftrightarrow \operatorname{IS}(A \cap B, A)=[1,1] \Leftrightarrow A \cap B=$ $A \Leftrightarrow A \subseteq B$.

(IP3) If $A \subseteq B \subseteq C$, then $\operatorname{IS}(A, C) \leq \operatorname{IS}(A, B), \operatorname{IS}(A, C) \leq$ IS $(B, C)$, and

$$
\begin{aligned}
& \mathrm{II}(C, A)=\operatorname{IS}(A \cap C, C)=\operatorname{IS}(A, C) ; \\
& \mathrm{II}(B, A)=\operatorname{IS}(A \cap B, B)=\operatorname{IS}(A, B) ; \\
& \mathrm{II}(C, B)=\operatorname{IS}(B \cap C, C)=\operatorname{IS}(B, C) .
\end{aligned}
$$

Namely, $\mathrm{II}(C, A) \leq \mathrm{II}(B, A), \mathrm{II}(C, A) \leq \mathrm{II}(C, B)$.

For example, if

$$
\begin{gathered}
\operatorname{IS}(A, B)=\left[\frac{1}{n} \sum_{i=1}^{n} \frac{\mu_{A}\left(x_{i}\right) \wedge \mu_{B}\left(x_{i}\right)}{\mu_{A}\left(x_{i}\right) \vee \mu_{B}\left(x_{i}\right)}, \frac{1}{n}\right. \\
\left.\cdot \sum_{i=1}^{n} \frac{2 \mu_{A}\left(x_{i}\right) \cdot \mu_{B}\left(x_{i}\right)}{\left(\mu_{A}\left(x_{i}\right)\right)^{2}+\left(\mu_{B}\left(x_{i}\right)\right)^{2}}\right],
\end{gathered}
$$

then

$$
\begin{gathered}
\operatorname{IE}_{13}(A)=\operatorname{IS}\left(A, A^{c}\right)=\left[\frac{1}{n} \sum_{i=1}^{n} \frac{\mu_{A}\left(x_{i}\right) \wedge \mu_{A}^{c}\left(x_{i}\right)}{\mu_{A}\left(x_{i}\right) \vee \mu_{A}^{c}\left(x_{i}\right)}, \frac{1}{n}\right. \\
\left.\cdot \sum_{i=1}^{n} \frac{2 \mu_{A}\left(x_{i}\right) \cdot \mu_{A}^{c}\left(x_{i}\right)}{\left(\mu_{A}\left(x_{i}\right)\right)^{2}+\left(\mu_{A}^{c}\left(x_{i}\right)\right)^{2}}\right]
\end{gathered}
$$

is an interval entropy. And

$$
\begin{gathered}
\operatorname{ID}_{12}(A, B)=\operatorname{IS}^{c}(A, B)=\left[\frac{1}{n}\right. \\
\cdot \sum_{i=1}^{n} \frac{\left(\mu_{A}\left(x_{i}\right)-\mu_{B}\left(x_{i}\right)\right)^{2}}{\left(\mu_{A}\left(x_{i}\right)\right)^{2}+\left(\mu_{B}\left(x_{i}\right)\right)^{2}}, \frac{1}{n} \\
\left.\cdot \sum_{i=1}^{n} \frac{\left|\mu_{A}\left(x_{i}\right)-\mu_{B}\left(x_{i}\right)\right|}{\mu_{A}\left(x_{i}\right) \vee \mu_{B}\left(x_{i}\right)}\right]
\end{gathered}
$$

is an interval distance measure. And

$$
\begin{aligned}
& \mathrm{II}_{7}(A, B)=\mathrm{IS}(B, A \cup B)=\left[\frac{1}{n}\right. \\
& \cdot \sum_{i=1}^{n} \frac{\mu_{B}\left(x_{i}\right)}{\mu_{A}\left(x_{i}\right) \vee \mu_{B}\left(x_{i}\right)}, \frac{1}{n} \\
& \left.\cdot \sum_{i=1}^{n} \frac{2 \mu_{B}\left(x_{i}\right) \cdot\left(\mu_{A}\left(x_{i}\right) \vee \mu_{B}\left(x_{i}\right)\right)}{\left(\mu_{B}\left(x_{i}\right)\right)^{2}+\left(\mu_{A}\left(x_{i}\right) \vee \mu_{B}\left(x_{i}\right)\right)^{2}}\right], \\
& \mathrm{II}_{8}(A, B)=\mathrm{IS}(A \cap B, A)=\left[\frac{1}{n}\right. \\
& \cdot \sum_{i=1}^{n} \frac{\mu_{A}\left(x_{i}\right) \wedge \mu_{B}\left(x_{i}\right)}{\mu_{A}\left(x_{i}\right)}, \frac{1}{n} \\
& \left.\cdot \sum_{i=1}^{n} \frac{2 \mu_{A}\left(x_{i}\right) \cdot\left(\mu_{A}\left(x_{i}\right) \wedge \mu_{B}\left(x_{i}\right)\right)}{\left(\mu_{A}\left(x_{i}\right)\right)^{2}+\left(\mu_{A}\left(x_{i}\right) \wedge \mu_{B}\left(x_{i}\right)\right)^{2}}\right]
\end{aligned}
$$

are interval inclusion measures.

Theorem 17. For $A, B \in F(X)$, ID is interval distance measure of fuzzy sets; then

(1) $I D^{c}(A, B)$ is interval similarity measure;

(2) $I D^{c}\left(A, A^{c}\right)$ is interval entropy;

(3) $I D^{c}(B, A \cup B), \operatorname{ID}^{c}(A \cap B, A)$ are interval inclusion measures.

Proof. (1) It can be easily concluded by Theorem 15.

(2) It can be easily concluded by Theorem 13 .

(3) It can be easily concluded by Theorems 13 and 15. For example, if

$$
\begin{gathered}
\operatorname{ID}(A, B)=\left[\frac{1}{n} \sum_{i=1}^{n}\left|\frac{\mu_{A}\left(x_{i}\right)-\mu_{B}\left(x_{i}\right)}{\mu_{A}\left(x_{i}\right)+\mu_{B}\left(x_{i}\right)}\right|, 1\right. \\
\left.-\frac{1}{n} \sum_{i=1}^{n} \frac{\mu_{A}\left(x_{i}\right) \wedge \mu_{B}\left(x_{i}\right)}{\mu_{A}\left(x_{i}\right) \vee \mu_{B}\left(x_{i}\right)}\right],
\end{gathered}
$$

then

$$
\begin{aligned}
& \operatorname{IS}_{12}(A, B)=\operatorname{ID}^{c}(A, B)=\left[\frac{1}{n} \sum_{i=1}^{n} \frac{\mu_{A}\left(x_{i}\right) \wedge \mu_{B}\left(x_{i}\right)}{\mu_{A}\left(x_{i}\right) \vee \mu_{B}\left(x_{i}\right)}, 1\right. \\
& \left.-\frac{1}{n} \sum_{i=1}^{n}\left|\frac{\mu_{A}\left(x_{i}\right)-\mu_{B}\left(x_{i}\right)}{\mu_{A}\left(x_{i}\right)+\mu_{B}\left(x_{i}\right)}\right|\right]
\end{aligned}
$$

is an interval similarity measure. And

$$
\begin{gathered}
\mathrm{II}_{14}(A)=\operatorname{ID}^{c}\left(A, A^{c}\right)=\left[\frac{1}{n} \sum_{i=1}^{n} \frac{\mu_{A}\left(x_{i}\right) \wedge \mu_{A}^{c}\left(x_{i}\right)}{\mu_{A}\left(x_{i}\right) \vee \mu_{A}^{c}\left(x_{i}\right)}, 1\right. \\
\left.-\frac{1}{n} \sum_{i=1}^{n}\left|\mu_{A}\left(x_{i}\right)-\mu_{A}^{c}\left(x_{i}\right)\right|\right]
\end{gathered}
$$


is an interval entropy. And

$$
\begin{aligned}
& \mathrm{II}_{9}(A, B)=\operatorname{ID}^{c}(B, A \cup B) \\
&= {\left[\frac{1}{n} \sum_{i=1}^{n} \frac{\mu_{B}\left(x_{i}\right)}{\mu_{A}\left(x_{i}\right) \vee \mu_{B}\left(x_{i}\right)}, 1\right.} \\
&-\left.\frac{1}{n} \sum_{i=1}^{n}\left|\frac{\mu_{B}\left(x_{i}\right)-\mu_{A}\left(x_{i}\right) \vee \mu_{B}\left(x_{i}\right)}{\mu_{B}\left(x_{i}\right)+\mu_{A}\left(x_{i}\right) \vee \mu_{B}\left(x_{i}\right)}\right|\right] \\
& \mathrm{II}_{10}(A, B)=\mathrm{ID}^{c}(A \cap B, A) \\
&=\left[\frac{1}{n} \sum_{i=1}^{n} \frac{\mu_{A}\left(x_{i}\right) \wedge \mu_{B}\left(x_{i}\right)}{\mu_{A}\left(x_{i}\right)}, 1\right. \\
&\left.-\frac{1}{n} \sum_{i=1}^{n}\left|\frac{\mu_{A}\left(x_{i}\right) \wedge \mu_{B}\left(x_{i}\right)-\mu_{A}\left(x_{i}\right)}{\mu_{A}\left(x_{i}\right) \wedge \mu_{B}\left(x_{i}\right)+\mu_{A}\left(x_{i}\right)}\right|\right]
\end{aligned}
$$

are interval inclusion measures.

Theorem 18. For $A, B \in F(X)$, II is interval inclusion measure of fuzzy sets; then

(1) $I I(A \cup B, A \cap B)$ is interval similarity measure;

(2) $\operatorname{II}\left(A \cup A^{c}, A \cap A^{c}\right)$ is interval entropy;

(3) $I I^{c}(A \cup B, A \cap B)$ is interval distance measure.

Proof. (1) Let $\operatorname{IS}(A, B)=\operatorname{II}(A \cup B, A \cap B)$.

(SP1) If $A \in P(X)$, then $\operatorname{IS}\left(A, A^{c}\right)=\operatorname{II}\left(A \cup A^{c}, A \cap A^{c}\right)=$ $\operatorname{II}(X, \phi)=[0,0]$.

$(\mathrm{SP} 2) \operatorname{IS}(A, A)=\operatorname{II}(A \cup A, A \cap A)=\operatorname{II}(A, A)=[1,1]$.

(SP3) It is obvious that $\operatorname{IS}(A, B)=\operatorname{IS}(B, A)$.

(SP4) If $A \subseteq B \subseteq C$, then $\mathrm{II}(C, A) \leq \mathrm{II}(B, A), \mathrm{II}(C, A) \leq$ $\mathrm{II}(C, B)$ and

$$
\begin{aligned}
& \operatorname{IS}(A, C)=\operatorname{IS}(A \cup C, A \cap C)=\mathrm{II}(C, A) ; \\
& \operatorname{IS}(A, B)=\operatorname{IS}(A \cup B, A \cap B)=\mathrm{II}(B, A) ; \\
& \operatorname{IS}(B, C)=\operatorname{IS}(B \cup C, B \cap C)=\mathrm{II}(C, B) .
\end{aligned}
$$

Namely,

$$
\begin{aligned}
& \operatorname{IS}(A, C) \leq \operatorname{IS}(A, B), \\
& \operatorname{IS}(A, C) \leq \operatorname{IS}(B, C) .
\end{aligned}
$$

(2) Let $\operatorname{IE}(A)=\operatorname{II}\left(A \cup A^{c}, A \cap A^{c}\right)$.

(EP1) If $A \in P(X)$, then $\operatorname{IE}(A)=\operatorname{II}\left(A \cup A^{c}, A \cap A^{c}\right)=$ $\mathrm{II}(X, \phi)=[0,0]$.

(EP2) If $\forall x \in X, \mu_{A}(x) \equiv 1 / 2$, then $A(x)=A^{c}(x)$ and

$$
\operatorname{IE}(A)=\operatorname{II}\left(A \cup A^{c}, A \cap A^{c}\right)=\operatorname{II}(A, A)=[1,1] .
$$

(EP3) When $\forall x \in X, \mu_{A_{2}}(x) \leq \mu_{A_{1}}(x) \leq 1 / 2$, we have $\mu_{A_{2}}(x) \leq \mu_{A_{1}}(x) \leq 1 / 2 \leq \mu_{A_{1}}^{c}(x) \leq \mu_{A_{2}}^{c}(x)$; then

$$
A_{2} \subseteq A_{1} \subseteq A_{1}^{c} \subseteq A_{2}^{c} .
$$

Namely,

$$
\begin{aligned}
& \operatorname{IE}\left(A_{1}\right)=\operatorname{II}\left(A_{1} \cup A_{1}^{c}, A_{1} \cap A_{1}^{c}\right)=\operatorname{II}\left(A_{1}^{c}, A_{1}\right), \\
& \operatorname{IE}\left(A_{2}\right)=\operatorname{II}\left(A_{2} \cup A_{2}^{c}, A_{2} \cap A_{2}^{c}\right)=\operatorname{II}\left(A_{2}^{c}, A_{2}\right) .
\end{aligned}
$$

For $\operatorname{II}\left(A_{2}^{c}, A_{2}\right) \leq \operatorname{II}\left(A_{2}^{c}, A_{1}\right) \leq \operatorname{II}\left(A_{1}^{c}, A_{1}\right)$, we have $\operatorname{IE}\left(A_{2}\right) \leq \operatorname{IE}\left(A_{1}\right)$.

When $\forall x \in X, \mu_{A_{2}}(x) \geq \mu_{A_{1}}(x) \geq 1 / 2$, the proof is similar.

(EP4) It is obvious that $\operatorname{IE}(A)=\operatorname{IE}\left(A^{c}\right)$.

(3) It can be easily concluded by Theorems 15 and 17. For example, if

$$
\begin{array}{r}
\mathrm{II}(A, B)=\left[\frac{1}{n} \sum_{i=1}^{n}\left(\frac{\mu_{A}\left(x_{i}\right) \wedge \mu_{B}\left(x_{i}\right)}{\mu_{A}\left(x_{i}\right)}\right)^{p}, \frac{1}{n}\right. \\
\left.\cdot \sum_{i=1}^{n}\left(\frac{\mu_{A}\left(x_{i}\right) \wedge \mu_{B}\left(x_{i}\right)}{\mu_{A}\left(x_{i}\right)}\right)^{1 / p}\right] \quad(p \geq 1),
\end{array}
$$

then

$$
\begin{aligned}
& \mathrm{IS}_{13}(A, B)=\mathrm{II}(A \cup B, A \cap B)=\left[\frac{1}{n}\right. \\
& \cdot \sum_{i=1}^{n}\left(\frac{\mu_{A}\left(x_{i}\right) \wedge \mu_{B}\left(x_{i}\right)}{\mu_{A}\left(x_{i}\right) \vee \mu_{B}\left(x_{i}\right)}\right)^{p}, \frac{1}{n} \\
& \left.. \sum_{i=1}^{n}\left(\frac{\mu_{A}\left(x_{i}\right) \wedge \mu_{B}\left(x_{i}\right)}{\mu_{A}\left(x_{i}\right) \vee \mu_{B}\left(x_{i}\right)}\right)^{1 / p}\right] \quad(p \geq 1)
\end{aligned}
$$

is an interval similarity measure. And

$$
\begin{gathered}
\operatorname{IE}_{15}(A, B)=\operatorname{II}\left(A \cup A^{c}, A \cap A^{c}\right)=\left[\frac{1}{n}\right. \\
\cdot \sum_{i=1}^{n}\left(\frac{\mu_{A}\left(x_{i}\right) \wedge \mu_{A}^{c}\left(x_{i}\right)}{\mu_{A}\left(x_{i}\right) \vee \mu_{A}^{c}\left(x_{i}\right)}\right)^{p}, \frac{1}{n} \\
\left.\cdot \sum_{i=1}^{n}\left(\frac{\mu_{A}\left(x_{i}\right) \wedge \mu_{A}^{c}\left(x_{i}\right)}{\mu_{A}\left(x_{i}\right) \vee \mu_{A}^{c}\left(x_{i}\right)}\right)^{1 / p}\right] \quad(p \geq 1)
\end{gathered}
$$

is an interval entropy. And

$$
\begin{aligned}
& \operatorname{ID}_{13}(A, B)=\mathrm{II}^{c}(A \cup B, A \cap B)=[1 \\
& -\frac{1}{n} \sum_{i=1}^{n}\left(\frac{\mu_{A}\left(x_{i}\right) \wedge \mu_{B}\left(x_{i}\right)}{\mu_{A}\left(x_{i}\right) \vee \mu_{B}\left(x_{i}\right)}\right)^{1 / p}, 1 \\
& \left.-\frac{1}{n} \sum_{i=1}^{n}\left(\frac{\mu_{A}\left(x_{i}\right) \wedge \mu_{B}\left(x_{i}\right)}{\mu_{A}\left(x_{i}\right) \vee \mu_{B}\left(x_{i}\right)}\right)^{p}\right] \quad(p \geq 1)
\end{aligned}
$$

is an interval distance measure.

Above all, it can be concluded that, among interval entropy, interval similarity measure, interval distance measure and interval inclusion measure, any two can be transformed into each other. 


\section{The Application to Fuzzy Multiple Attribute Decision Making}

Given $m$ alternatives and $n$ criteria, a typical FMADM problem can be expressed in matrix format as

$$
\widetilde{X}=\begin{gathered}
C_{1} \\
A_{1} \\
\vdots \\
A_{m}
\end{gathered}\left[\begin{array}{ccc}
\tilde{x}_{11} & \cdots & \tilde{x}_{1 n} \\
\vdots & \vdots & \vdots \\
\tilde{x}_{m 1} & \cdots & \tilde{x}_{m n}
\end{array}\right],
$$

where $A_{1}, A_{2}, \ldots, A_{m}$ are the alternatives to be chosen, $C_{1}, C_{2}, \ldots, C_{n}$ denote the evaluation criteria, $\tilde{x}_{i j}$ represents the value of alternative $A_{i}$ with respect to criterion $C_{j}$ evaluated, and the set $W=\left\{\omega_{1}, \omega_{2}, \ldots, \omega_{n}\right\}$ is the weight of criterion $[4-6,8,9,11,12]$. Then the alternatives can be ranked based on the given information [36-38]. In this section, the steps of the proposed method are described with an example. How to determine the weights of all alternatives is described in Section 5.1. And the method to compute the evaluation values of all alternatives with respect to criteria is listed in Section 5.2. The method to rank the order of all alternatives named as possibility-based comparison relation is expressed in Section 5.3. Finally, a FMADM problem with thirty-one alternatives and twenty-three criteria is used to explain the process of the decision making.

5.1. Internal Entropy Weight. It is well known that entropy is a method in obtaining the weights for a MADM problem especially when obtaining suitable weights based on the preferences and decision making experiments are not possible [22]. The original procedure of entropy weight can be expressed in a series of steps [39]:

(1) Compute entropy $E_{j}$.

(2) Set $\omega_{j}=\left(1-E_{j}\right) / E, E=\sum_{s=1}^{n}\left(1-E_{s}\right), j=1,2, \ldots, n$, as the weight of attribute $j$.

Now suppose that the weight of the elements of decision matrix is difficult and uncertain, as a result, their values are considered as intervals. Entropy can be extended to interval entropy. The steps are as follows:

(I1) Compute interval entropy $\mathrm{IE}_{i j}=\left[\mathrm{IE}_{i j}^{L}, \mathrm{IE}_{i j}^{R}\right], i=1,2$, $\ldots, m, j=1,2, \ldots, n$.

(I2) Set $\omega_{i j}=\left[\omega_{i j}^{L}, \omega_{i j}^{R}\right]=\left[1-\mathrm{IE}_{i j}^{R}, 1-\mathrm{IE}_{i j}^{L}\right], i=1,2, \ldots, m$, $j=1,2, \ldots, n$, as the weight of attribute $j$.

For instance, if $A=[0.5,0.6,0.7,0.8]$, by (7), then interval entropy and interval entropy weight can be shown as $[0.5,0.6667]$ and $[0.3333,0.5]$, respectively.

5.2. The Evaluation Values Obtained by the Weight Average. To the given $\alpha$ value, the $\alpha$-cut sets of the weight $\omega_{j}$ and the alternatives $\tilde{x}_{i j}$ can be denoted by $\left(\omega_{j}\right)_{\alpha}=\left[\left(\omega_{j}\right)_{\alpha}^{L},\left(\omega_{j}\right)_{\alpha}^{R}\right]$ and $\left(\tilde{x}_{i j}\right)_{\alpha}=\left[\left(\tilde{x}_{i j}\right)_{\alpha}^{L},\left(\tilde{x}_{i j}\right)_{\alpha}^{R}\right]$, respectively. So the evaluation values of the given $\alpha$ value can be denoted by the following:

$$
\begin{aligned}
\left(F_{i}\right)_{\alpha} & =\left[\left(F_{i}\right)_{\alpha}^{L},\left(F_{i}\right)_{\alpha}^{R}\right] \\
& =\left[\sum_{j=1}^{n}\left(\omega_{j}\right)_{\alpha}^{L}\left(x_{i j}\right)_{\alpha}^{L}, \sum_{j=1}^{n}\left(\omega_{j}\right)_{\alpha}^{R}\left(x_{i j}\right)_{\alpha}^{R}\right] .
\end{aligned}
$$

Remark 19 (see [40]). Here, two reasons are responded to the unnormalization. Firstly, the evaluation values after normalization are not always in $[0,1]$. Secondly, the normalization can only effect the proportion changes of the evaluation values, without any changes to the actual ranking results.

For instance, if $A=[0.5,0.6,0.7,0.8]$, then interval entropy weight can be shown as $[0.3333,0.5]$ and the $\alpha$-cut sets can be denoted by

$$
\begin{aligned}
& A_{\alpha} \\
& = \begin{cases}{[0.6 \lambda+0.5(1-\lambda), 0.7 \lambda+0.8(1-\lambda)]} & \lambda \in(0,1) \\
{[0.6,0.7]} & \lambda=1 .\end{cases}
\end{aligned}
$$

So

$$
F_{\alpha}=[0.05556 \alpha+0.2778,0.53336-0.06667 \alpha] .
$$

5.3. The Possibility-Based Method for Ranking Fuzzy Numbers [41]. In this section, possibility-based comparison relation is presented. Since the $\alpha$-cut sets of fuzzy numbers can be expressed as the interval value sets, possibility-based comparison relation on interval values is proposed in Section 5.3.1, and then the comparison relation on fuzzy numbers is presented in Section 5.3.2.

5.3.1. Interval Values. Assuming $X=\left[x^{-}, x^{+}\right], Y=\left[y^{-}, y^{+}\right]$ are the interval values, $X_{\lambda}, Y_{\lambda}(\lambda \in R)$ can be constructed as follows:

$$
\begin{aligned}
& X_{\lambda}= \begin{cases}1 & x^{-} \geq \lambda \\
\frac{1}{2} & x^{-}<\lambda \leq x^{+} \\
0 & x^{+}<\lambda,\end{cases} \\
& Y_{\alpha}= \begin{cases}1 & y^{-} \geq \lambda \\
\frac{1}{2} & y^{-}<\lambda \leq y^{+} \\
0 & y^{+}<\lambda .\end{cases}
\end{aligned}
$$

Set

$$
G(\lambda)= \begin{cases}1 & X_{\lambda}>Y_{\lambda} \\ 0 & X_{\lambda} \leq Y_{\lambda}\end{cases}
$$

$P(X>Y)$ is used to represent the degree of interval values " $X>Y$ " which is named as possibility-based comparison relation on interval values:

$$
P(X>Y)=\frac{1}{x^{+} \vee y^{+}-x^{-} \wedge y^{-}} \int_{x^{-} \wedge y^{-}}^{x^{+} \vee y^{+}} G(\lambda) d \lambda .
$$


TABLE 1: The ranking results.

\begin{tabular}{|c|c|c|c|}
\hline & $A=[0,0.1,0.5,1]$ & $B=[0.3,0.5,0.8,0.9]$ & Ranking order \\
\hline Optimistic & 0 & 0.065 & $A \prec B$ \\
\hline Neutral & 0.5 & 0.4 & $A \prec B$ \\
\hline Pessimistic & 0.4547 & 0.7723 & $A \prec B$ \\
\hline
\end{tabular}

5.3.2. Fuzzy Numbers. If $A$ and $B$ are two fuzzy numbers, then the possibility-based comparison relation between fuzzy numbers can be denoted by $P(A>B)$ as follows:

$$
P(A>B)=\int_{0}^{1} P\left(A_{\alpha}>B_{\alpha}\right) d \alpha .
$$

Let $S=\left\{S_{1}, S_{2}, \ldots, S_{n}\right\}$ be a set which contains finite fuzzy numbers to be ranked; we can select function $T$ as the evaluation of comparisons [42]. Then the fuzzy numbers can be ranked as the values of $P\left(S_{i}>T\right)$. Here, $T$ can be seen as an attitude which comes from the decision maker. Usually, the attitude of the decision maker can be classified into three groups: neutral, optimistic, and pessimistic. The optimistic attitude reflects the preferences of decision makers to the maximal payoff, and it is often used as follows:

$$
\mu_{A_{\text {opt }}}(x)= \begin{cases}\frac{x-x_{\min }}{x_{\max }-x_{\min }} & x_{\min } \leq x \leq x_{\max } \\ 0 & \text { else. }\end{cases}
$$

The neutral attitude reflects the preferences of decision makers for not only the payoff which could be obtained but also the risk which could be faced, and it is often used as follows:

$$
\mu_{A_{\text {neu }}}(x)= \begin{cases}1 & x_{\min } \leq x \leq x_{\max } \\ 0 & \text { else. }\end{cases}
$$

And the last attitude is the pessimistic one reflecting the preferences of decision makers for not only the payoff which could be gained but also the risk which could be faced. It is often used as follows:

$$
\mu_{A_{\text {pess }}}(x)= \begin{cases}\frac{x_{\max }-x}{x_{\max }-x_{\min }} & x_{\min } \leq x \leq x_{\max } \\ 0 & \text { else. }\end{cases}
$$

For example, the ranking results with respect to three attitudes are shown in Table 1.

5.4. A Numerical Example. In this section, the steps of the proposed method are described with an example. Suppose that there is a FMADM problem with thirty-one alternatives and twenty-three criteria. Data are presented in Tables 2-3 [43] which can be represented as seven linguistic variables to evaluate degree of impact for railway reconstruction projects and their corresponding trapezoid fuzzy numbers are listed in Table 4 . The decision making can be conducted as follows.

\begin{tabular}{|c|c|c|c|c|c|c|c|c|c|c|c|c|c|}
\hline & & $C_{1}$ & $C_{2}$ & $C_{3}$ & 4 & $C_{5}$ & $C_{6}$ & $C_{7}$ & $C_{8}$ & $C_{9}$ & $C_{10}$ & $C_{11}$ & $C_{12}$ \\
\hline \multirow{4}{*}{$A_{1}$} & 11 & 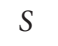 & $S$ & $V S$ & VS & $A$ & $A$ & $A$ & $U$ & $S$ & $A$ & $U$ & 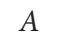 \\
\hline & $A_{12}$ & $A$ & $A S$ & $A S$ & $A S$ & $V S$ & $A$ & $V S$ & $A$ & $V S$ & $S$ & $S$ & $V S$ \\
\hline & $A_{13}$ & $V S$ & $V S$ & $V S$ & $V S$ & $S$ & $V S$ & $S$ & $S$ & $A S$ & $S$ & $V S$ & $V S$ \\
\hline & $\mathrm{A}_{14}$ & $V S$ & $V S$ & $V S$ & $A$ & A & $S$ & $A$ & $A$ & $S$ & $U$ & A & $V S$ \\
\hline \multirow{4}{*}{$A_{2}$} & & $A$ & 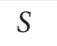 & 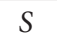 & $S$ & 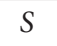 & $\Lambda$ & 11 & $V S$ & $A$ & & $S$ & $\sigma$ \\
\hline & $A_{22}$ & $A$ & $A$ & $S$ & $V S$ & $S$ & $U$ & $A$ & $S$ & 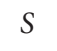 & $U$ & 0 & S \\
\hline & $A_{23}$ & $A$ & $A$ & $S$ & $S$ & $A$ & $U$ & $A$ & $V S$ & $A$ & 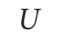 & $S$ & 0 \\
\hline & $A_{24}$ & $S$ & $A$ & $S$ & $A S$ & $A$ & $A$ & $S$ & $S$ & $A$ & $A$ & $V S$ & 5 \\
\hline \multirow{4}{*}{$A_{3}$} & $I_{31}$ & $V S$ & $U$ & $A S$ & $A S$ & $V S$ & $S$ & $A$ & $A S$ & $A$ & 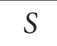 & $S$ & $S$ \\
\hline & $A_{32}$ & $A$ & $U$ & $V S$ & $S$ & $S$ & S & $A$ & $V S$ & $A$ & $A$ & $S$ & $V S$ \\
\hline & $A_{33}$ & $A$ & $A$ & $S$ & $V S$ & $S$ & $A$ & $U$ & $V S$ & $A$ & 1 & $A$ & $V S$ \\
\hline & $A_{34}$ & $S$ & $A$ & $V S$ & $A$ & VS & $A$ & A & $A S$ & $S$ & A & $S$ & $\mathrm{~J}$ \\
\hline \multirow{6}{*}{$A$} & & $U$ & $V S$ & $V S$ & $A S$ & $V S$ & $S$ & $A$ & $A S$ & $A$ & VS & $V S$ & $A S$ \\
\hline & $A_{42}$ & $A$ & $V S$ & $A$ & $S$ & $S$ & $S$ & $S$ & $V S$ & $V S$ & & $A$ & $S$ \\
\hline & $A_{43}$ & $A$ & $V S$ & $S$ & IS & 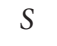 & $A$ & $A$ & $S$ & 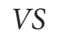 & & $S$ & \\
\hline & $A_{44}$ & $A$ & $S$ & VC & $S$ & $V S$ & $\mathrm{~s}$ & 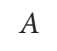 & $S$ & $A$ & & $S$ & \\
\hline & $A_{45}$ & $A$ & $V S$ & AD & $A S$ & VS & 3 & 0 & $S$ & $A$ & $\Lambda$ & $A$ & $A \Delta$ \\
\hline & $A_{46}$ & $A$ & $S$ & $V S$ & 3 & $V S$ & $S$ & $V S$ & $A S$ & $A$ & A & $S$ & $V S$ \\
\hline \multirow{4}{*}{$A_{5}$} & $I_{51}$ & $S$ & $U$ & $A$ & $V S$ & $S$ & $V S$ & $S$ & $A S$ & $V S$ & 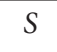 & $U$ & $A S$ \\
\hline & $A_{52}$ & $V S$ & $A$ & $S$ & $S$ & $A$ & $A$ & $A$ & $S$ & $S$ & $A$ & $S$ & $A S$ \\
\hline & $A_{53}$ & $V S$ & $A$ & $S$ & $U$ & $A$ & $A$ & $A$ & $A$ & $c$ & $A$ & $U$ & S \\
\hline & $A_{54}$ & $S$ & $S$ & $S$ & $A$ & $S$ & $S$ & A & $V S$ & $A S$ & & $S$ & $V S$ \\
\hline \multirow{5}{*}{$A_{6}$} & $A_{61}$ & $A$ & $S$ & $A S$ & $V S$ & $V S$ & $S$ & u & $S$ & - & $\Lambda$ & $A$ & 3 \\
\hline & $A_{62}$ & $A$ & $S$ & $V S$ & $S$ & $S$ & $S$ & $\mathrm{~J}$ & $S$ & 3 & $A$ & $A$ & 3 \\
\hline & $A_{63}$ & $A$ & $V S$ & $V S$ & $S$ & $S$ & $A$ & $A$ & $A$ & $A$ & $A$ & $A$ & $S$ \\
\hline & $A_{64}$ & $A$ & $V S$ & $V S$ & $A$ & $S$ & $S$ & $S$ & $V S$ & $A$ & $A$ & $A$ & $S$ \\
\hline & $A_{65}$ & $A$ & $V S$ & $A S$ & $V S$ & $S$ & $S$ & $A$ & $A S$ & $A$ & $S$ & $S$ & $V S$ \\
\hline \multirow{4}{*}{17} & $A_{71}$ & $A$ & $S$ & $A S$ & 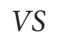 & $S$ & $A$ & $S$ & $U$ & $A$ & $A$ & $A$ & $A$ \\
\hline & $A_{72}$ & $A$ & $V S$ & $A S$ & $A S$ & $S$ & $A$ & $V S$ & $A S$ & $A$ & $S$ & $A$ & $S$ \\
\hline & $A_{73}$ & $U$ & $A$ & $A S$ & $S$ & $V S$ & $U$ & $S$ & $S$ & $A$ & $A$ & $A$ & VS \\
\hline & $A_{74}$ & $U$ & $U$ & $A S$ & $S$ & $S$ & $A$ & $A$ & $S$ & $S$ & $S$ & $S$ & $V S$ \\
\hline
\end{tabular}

Step 1. Compute interval entropy weight of all alternatives according to the corresponding the $\alpha$-cut sets. Here, to
TABLE 2: The evaluation values of $C_{1}-C_{12}$.

the trapezoid fuzzy number $[a, b, c, d]$, the interval entropy $\mathrm{IE}_{7}(A)$ can be expressed as

$$
\mathrm{IE}_{7}(A)=\left[\frac{b-a-c+d}{2(d-a)}, \frac{2(b-a-c+d)}{3(d-a)}\right]
$$

Then the corresponding $\alpha$-cut sets and interval entropy weights are listed in Table 5.

Step 2. Compute the evaluation values.

The alternative values $\left(F_{i}\right)_{\alpha}$ can be drawn by (99) and the results are listed in Table 6.

Step 3. Ranking of all alternatives: the alternatives can be ranked by (105). 
TABLE 3: The evaluation values of $C_{12}-C_{23}$.

\begin{tabular}{|c|c|c|c|c|c|c|c|c|c|c|c|c|}
\hline & & $C_{13}$ & $C_{14}$ & $C_{15}$ & $C_{16}$ & $C_{17}$ & $C_{18}$ & $C_{19}$ & $C_{20}$ & $C_{21}$ & $C_{22}$ & $C_{23}$ \\
\hline \multirow{4}{*}{$A_{1}$} & $A_{11}$ & $A$ & $S$ & $A$ & $S$ & $A$ & $S$ & $V S$ & $A$ & $A$ & $S$ & $S$ \\
\hline & $A_{12}$ & $S$ & $A S$ & $A$ & $S$ & $U$ & $S$ & $V S$ & $A S$ & $S$ & $U$ & $V S$ \\
\hline & $A_{13}$ & $V S$ & $V S$ & $S$ & $V S$ & $V S$ & $S$ & $V S$ & $S$ & $V S$ & $S$ & $S$ \\
\hline & $A_{14}$ & $U$ & $A$ & $S$ & $A$ & $A$ & $A$ & $S$ & A & $U$ & $A$ & $S$ \\
\hline \multirow{4}{*}{$A_{2}$} & $A_{21}$ & $V S$ & $S$ & $U$ & $S$ & $V S$ & $V S$ & $A$ & $S$ & $A$ & $A$ & $S$ \\
\hline & $A_{22}$ & $V S$ & $V S$ & $A$ & $S$ & $S$ & $S$ & $S$ & $A S$ & $U$ & $A$ & $V S$ \\
\hline & $A_{23}$ & $S$ & $A$ & $U$ & $A$ & $S$ & $V S$ & $S$ & $S$ & $V U$ & $A$ & $S$ \\
\hline & $A_{24}$ & $V S$ & $S$ & $U$ & $S$ & $S$ & $S$ & $S$ & $V S$ & A & $A$ & $V S$ \\
\hline \multirow{4}{*}{$A_{3}$} & $A_{31}$ & $V S$ & $V S$ & $A$ & $S$ & $A$ & $S$ & $A$ & $S$ & $U$ & $A$ & $V S$ \\
\hline & $A_{32}$ & $V S$ & $S$ & $S$ & $S$ & $A$ & $S$ & $S$ & $S$ & $S$ & $A$ & $S$ \\
\hline & $A_{33}$ & $S$ & $S$ & $A$ & $S$ & $A$ & $S$ & $S$ & $V S$ & $A$ & $U$ & $S$ \\
\hline & $A_{34}$ & $V S$ & $V S$ & $A$ & $S$ & $A$ & $S$ & $A$ & $S$ & $V S$ & $A$ & $S$ \\
\hline \multirow{6}{*}{$A_{4}$} & $A_{41}$ & $A S$ & $V S$ & $A$ & $A$ & $A$ & $S$ & $U$ & $V S$ & $V S$ & $U$ & $V S$ \\
\hline & $A_{42}$ & $S$ & $V S$ & $S$ & $S$ & $A$ & $S$ & $A$ & $V S$ & $S$ & $S$ & $S$ \\
\hline & $A_{43}$ & $V S$ & $S$ & $S$ & $S$ & $A$ & $S$ & $A S$ & $S$ & $A S$ & $A$ & $S$ \\
\hline & $A_{44}$ & $S$ & $V S$ & $U$ & $A$ & $A$ & $S$ & $U$ & $S$ & $A$ & $U$ & $S$ \\
\hline & $A_{45}$ & $A S$ & $A S$ & $U$ & $A$ & $A$ & $V S$ & $U$ & $V S$ & $S$ & $V U$ & $S$ \\
\hline & $A_{46}$ & VS & $V S$ & $S$ & $A$ & $A$ & $V S$ & $A$ & $S$ & $A$ & $V U$ & VS \\
\hline \multirow{4}{*}{$A_{5}$} & $A_{51}$ & $A S$ & $V S$ & $U$ & $S$ & $S$ & $S$ & $V U$ & $V S$ & $V U$ & $S$ & $S$ \\
\hline & $A_{52}$ & $V S$ & $V S$ & $U$ & $S$ & $S$ & $S$ & $V U$ & $S$ & $A U$ & $S$ & $S$ \\
\hline & $A_{53}$ & $A$ & $S$ & $U$ & $A$ & $S$ & $A$ & $U$ & $S$ & $V U$ & $S$ & $S$ \\
\hline & $A_{54}$ & VS & $V S$ & $A$ & $A$ & $S$ & $S$ & $V U$ & $V S$ & $V U$ & $S$ & $S$ \\
\hline \multirow{5}{*}{$A_{6}$} & $A_{61}$ & $A S$ & $V S$ & $A$ & $S$ & $S$ & $V S$ & $A$ & $A S$ & $U$ & $A$ & $S$ \\
\hline & $A_{62}$ & VS & $S$ & $U$ & $A$ & $S$ & $S$ & $V U$ & $A$ & $S$ & $A$ & $S$ \\
\hline & $A_{63}$ & $V S$ & $V S$ & $A$ & $S$ & $S$ & $S$ & $V U$ & $S$ & $A S$ & $A$ & $V S$ \\
\hline & $A_{64}$ & $A S$ & $V S$ & $U$ & $A$ & $S$ & $S$ & $V U$ & $V S$ & $V U$ & $A$ & VS \\
\hline & $A_{65}$ & $A S$ & $A S$ & $U$ & $A$ & $S$ & $S$ & $S$ & $A S$ & $S$ & $S$ & $V S$ \\
\hline \multirow{4}{*}{$A_{7}$} & $A_{71}$ & $A S$ & $S$ & $A$ & $A$ & $V S$ & $A$ & $S$ & $S$ & $V U$ & $V S$ & $S$ \\
\hline & $A_{72}$ & $A$ & $V S$ & $U$ & $S$ & $V S$ & $A$ & $V U$ & $V S$ & $A U$ & $V S$ & $S$ \\
\hline & $A_{73}$ & $A$ & $S$ & $A$ & $A$ & $V S$ & $A$ & $V U$ & $V S$ & $A U$ & $V S$ & $S$ \\
\hline & $A_{74}$ & $V S$ & $S$ & $A$ & $A$ & $V S$ & $S$ & $U$ & $S$ & $U$ & $A$ & $S$ \\
\hline
\end{tabular}

TABLE 4: Semantic scale and corresponding trapezoidal fuzzy numbers.

\begin{tabular}{lc}
\hline Linguistic variables & Trapezoidal fuzzy numbers \\
\hline$A S$ (absolutely serious) & {$[0.8,0.9,1,1]$} \\
$V S$ (very serious) & {$[0.7,0.8,0.8,0.9]$} \\
$S$ (serious) & {$[0.5,0.6,0.7,0.8]$} \\
$A$ (average) & {$[0.4,0.5,0.5,0.6]$} \\
$U$ (unserious) & {$[0.2,0.3,0.4,0.5]$} \\
$V U$ (very unserious) & {$[0.1,0.2,0.2,0.3]$} \\
$A U$ (absolutely unserious) & {$[0.0,0.0,0.1,0.2]$} \\
\hline
\end{tabular}

Remark 20 (Lu's method). Lu's method is as follows:

(1) The linguistic variables are transferred into corresponding trapezoid fuzzy numbers:

$$
\widetilde{x}_{j}=\frac{1}{m} \sum_{i=1}^{m} \widetilde{x}_{i j}
$$

TABLE 5: The $\alpha$-cut sets of the linguistic variables and the corresponding interval entropy weights.

\begin{tabular}{lcc}
\hline $\begin{array}{l}\text { Linguistic } \\
\text { variables }\end{array}$ & $\alpha$-cut sets & Weights \\
\hline$A S$ & {$[0.9 \alpha+0.8(1-\alpha), 1]$} & {$[0.6667,0.75]$} \\
$V S$ & {$[0.8 \alpha+0.7(1-\alpha), 0.8 \alpha+0.9(1-\alpha)]$} & {$[0.3333,0.5]$} \\
$S$ & {$[0.6 \alpha+0.5(1-\alpha), 0.7 \alpha+0.8(1-\alpha)]$} & {$[0.5556,0.6667]$} \\
$A$ & {$[0.5 \alpha+0.4(1-\alpha), 0.5 \alpha+0.6(1-\alpha)]$} & {$[0.3333,0.5]$} \\
$U$ & {$[0.3 \alpha+0.2(1-\alpha), 0.4 \alpha+0.5(1-\alpha)]$} & {$[0.5556,0.6667]$} \\
$V U$ & {$[0.2 \alpha+0.1(1-\alpha), 0.2 \alpha+0.3(1-\alpha)]$} & {$[0.3333,0.5]$} \\
$A U$ & {$[0,0.1 \alpha+0.2(1-\alpha)]$} & {$[0.6667,0.75]$} \\
\hline
\end{tabular}

(2) Defuzzify each aggregated trapezoid fuzzy number into a crisp value:

$$
x_{j}=\frac{\int_{x \in U} x \mu_{\tilde{x}_{j}}(x) d x}{\int_{x \in U} \mu_{\tilde{x}_{j}}(x) d x} .
$$


TABLE 6: The evaluation values of all alternatives.

\begin{tabular}{|c|c|}
\hline$\left(F_{11}\right)_{\alpha}$ & {$[0.98889 \alpha+4.4777,29.28358-1.3167 \alpha]$} \\
\hline$\left(F_{12}\right)_{\alpha}$ & {$[1.11113 \alpha+6.48898,11.51686-1.03336 \alpha]$} \\
\hline$\left(F_{13}\right)_{\alpha}$ & {$[\alpha+6.06659,11.40024-1.25003 \alpha]$} \\
\hline$\left(F_{41}\right)_{\alpha}$ & {$[0.94443 \alpha+4.12212,8.76685-1.28336 \alpha]$} \\
\hline$\left(F_{21}\right)_{\alpha}$ & {$[1.03335 \alpha+4.86672,9.9003-1.35004 \alpha]$} \\
\hline$\left(F_{22}\right)_{\alpha}$ & {$[1.08892 \alpha+5.24456,10.38365-1.31671 \alpha]$} \\
\hline$\left(F_{23}\right)_{\alpha}$ & {$[1.03335 \alpha+4.40007,9.25029-1.35004 \alpha]$} \\
\hline$\left(F_{24}\right)_{\alpha}$ & {$[1.04446 \alpha+5.28896,10.31695-1.28337 \alpha]$} \\
\hline$\left(F_{31}\right)_{\alpha}$ & {$[1.06668 \alpha+5.73339,10.70022-1.15003 \alpha]$} \\
\hline$\left(F_{32}\right)_{\alpha}$ & {$[1.05558 \alpha+5.17788,10.33367-1.36671 \alpha]$} \\
\hline$\left(F_{33}\right)_{\alpha}$ & {$[0.96666 \alpha+4.53327,9.35022-1.30003 \alpha]$} \\
\hline$\left(F_{34}\right)_{\alpha}$ & {$[0.97777 \alpha+5.12219,9.96688-1.23336 \alpha]$} \\
\hline$\left(F_{41}\right)_{\alpha}$ & {$[1.0111 \alpha+5.78879,10.61677-1.03335 \alpha]$} \\
\hline$\left(F_{42}\right)_{\alpha}$ & {$[1.05558 \alpha+5.44455,10.68368-1.36671 \alpha]$} \\
\hline$\left(F_{43}\right)_{\alpha}$ & {$[1.10003 \alpha+6.0016,11.20032-1.25004 \alpha]$} \\
\hline$\left(F_{44}\right)_{\alpha}$ & {$[1.05558 \alpha+4.74453,9.78365-1.36671 \alpha]$} \\
\hline$\left(F_{45}\right)_{\alpha}$ & {$[1.10001 \alpha+6.30009,11.05014-0.95002 \alpha]$} \\
\hline$\left(F_{46}\right)_{\alpha}$ & {$[0.93331 \alpha+5.03321,9.80016-1.20002 \alpha]$} \\
\hline$\left(F_{51}\right)_{\alpha}$ & {$[1.13337 \alpha+5.80017,10.90029-1.20004 \alpha]$} \\
\hline$\left(F_{52}\right)_{\alpha}$ & {$[1.03336 \alpha+5.10014,10.10031-1.32504 \alpha]$} \\
\hline$\left(F_{53}\right)_{\alpha}$ & {$[1.03335 \alpha+4.1334,8.90028-1.35004 \alpha]$} \\
\hline$\left(F_{54}\right)_{\alpha}$ & {$[1.04446 \alpha+5.35565,10.36696-1.28337 \alpha]$} \\
\hline$\left(F_{61}\right)_{\alpha}$ & {$[1.08891 \alpha+5.94456,10.98359-1.1667 \alpha]$} \\
\hline$\left(F_{62}\right)_{\alpha}$ & {$[1.07781 \alpha+5.02239,10.11703-1.38338 \alpha]$} \\
\hline$\left(F_{63}\right)_{\alpha}$ & {$[0.95554 \alpha+4.87772,9.58352-1.21669 \alpha]$} \\
\hline$\left(F_{64}\right)_{\alpha}$ & {$[0.95554 \alpha+4.71104,9.38351-1.21669 \alpha]$} \\
\hline$\left(F_{65}\right)_{\alpha}$ & {$[1.15559 \alpha+6.74464,11.88359-1.0667 \alpha]$} \\
\hline$\left(F_{71}\right)_{\alpha}$ & {$[1.01111 \alpha+5.05558,9.76687-1.18336 \alpha]$} \\
\hline$\left(F_{72}\right)_{\alpha}$ & {$[0.96666 \alpha+5.33331,10.05015-1.12502 \alpha]$} \\
\hline$\left(F_{73}\right)_{\alpha}$ & {$[0.92221 \alpha+4.41104,9.0335-1.24169 \alpha]$} \\
\hline$\left(F_{74}\right)_{\alpha}$ & {$[1.11115 \alpha+5.12237,10.267-1.33338 \alpha]$} \\
\hline
\end{tabular}

(3) Normalize the degree of impact of risk factors for each risk aspect level:

$$
R_{j}=\frac{x_{j}}{\sum_{j=1}^{n} x_{j}} .
$$

The results of local ranking and global ranking under different attitudes are listed in Tables 7 and 8, respectively. And there are some different results with Lu's method. The values of all linguistic variables are determined by interval entropy weight. Here, the values are the most in $A S$ and $A U$, the following are $S$ and $U$, and then the least are $V S, A$, and $V U$. Among all these linguistic variables, $U, V U$, and $A U$ are inclined to the optimistic attitude, and $S, V S$, and $A S$ are inclined to the opposite. And the numbers of all linguistic variables are listed in Table 9. For example, to the second category, the numbers of $U$ and $A$ in $F_{21}$ and $F_{23}$ are 2 and 3 , respectively. So, for the optimistic attitude, the decision maker would prefer $F_{23}$ rather than $F_{21}$. The explanations to other cases are similar.

\section{Conclusions}

It is well known that entropy is a famous method in obtaining the weights for a MADM problem especially when obtaining suitable weights based on the preferences and decision making experiments are not possible. And most of the literatures pertaining to MADM analysis have been published using entropy weights. Among all these literatures, the result of entropy weight can be expressed as the certain number. But, in practice, because of uncertainty of people's cognition, when the weight of the alternatives cannot be measured precisely and be expressed as some uncertain types of data, how to use entropy to represent it is worthy of discussion. In this paper, the concept of interval entropy is proposed and the relationships among other conceptions such as interval entropy, interval similarity measure, interval distance measure, and interval inclusion measure are investigated in detail. And then, a FMADM model based on interval entropy is set up. In this model, interval entropy is used as the weight, by which the evaluation values can be obtained. And all alternatives with respect to each criterion can be ranked using the method based on the possibility degree. The achievements in the presented work will provide a new model for FMADM based on interval entropy weight. Because of the relationship between the interval entropy, interval similarity measure, interval distance measure, and inclusion measure, in the future, other types of uncertain weights will be researched in detail.

\section{Appendices}

\section{A. Entropy of Fuzzy Sets}

The entropy of fuzzy sets is a measure of fuzziness of the fuzzy sets. In 1972, Luca and Termini [44] introduced the axiom constructions of entropy of fuzzy sets: real function $E: F(X) \rightarrow[0,1] A \mapsto E(A)$ is called entropy on $F(X)$, if $E$ has the following properties:

(E1) If $A \in P(X)$, then $E(A)=0$.

(E2) If $\forall x \in X, \mu_{A}(x) \equiv 1 / 2$, then $E(A)=1$.

(E3) If $\forall x \in X, \mu_{A_{2}}(x) \leq \mu_{A_{1}}(x) \leq 1 / 2$ or $\mu_{A_{2}}(x) \geq$ $\mu_{A_{1}}(x) \geq 1 / 2$, then

$$
E\left(A_{2}\right) \leq E\left(A_{1}\right) .
$$

(E4) $E(A)=E\left(A^{c}\right)$.

For instance, if $X=\left\{x_{1}, x_{2}, \ldots, x_{n}\right\}$ and $A$ is a fuzzy set, then

$$
E(A)=\frac{1}{n} \sum_{i=1}^{n} \frac{\left(\mu_{A}\left(x_{i}\right) \wedge \mu_{A}^{c}\left(x_{i}\right)\right)}{\left(\mu_{A}\left(x_{i}\right) \vee \mu_{A}^{c}\left(x_{i}\right)\right)}
$$

is entropy of fuzzy set $A$.

\section{B. Similarity Measure of the Fuzzy Sets}

To indicate the degree of similarity of two fuzzy sets, the concept of similarity measure was proposed in 1993 [45]. 
TABLE 7: The local ranking order.

\begin{tabular}{|c|c|c|c|c|c|c|c|c|}
\hline & & Opt. & $\begin{array}{c}\text { Local ranking } \\
\text { order }\end{array}$ & Pess. & $\begin{array}{c}\text { Local ranking } \\
\text { order }\end{array}$ & Neu. & $\begin{array}{c}\text { Local ranking } \\
\text { order }\end{array}$ & $\begin{array}{c}\text { Lu local } \\
\text { ranking order }\end{array}$ \\
\hline \multirow{4}{*}{1} & $F_{11}$ & 0.001224 & 3 & 1.2839 & 3 & 0.1095 & 3 & 3 \\
\hline & $F_{12}$ & 0.06217 & 1 & 1.41998 & 2 & 0.3765 & 1 & 2 \\
\hline & $F_{13}$ & 0.04008 & 2 & 1.4397 & 1 & 0.3149 & 2 & 1 \\
\hline & $F_{41}$ & 0 & 4 & 1.25 & 4 & 0.0608 & 4 & 4 \\
\hline \multirow{4}{*}{2} & $F_{21}$ & 0.0055 & 4 & 1.3316 & 3 & 0.1625 & 3 & 3 \\
\hline & $F_{22}$ & 0.01291 & 2 & 1.3638 & 1 & 0.2148 & 2 & 2 \\
\hline & $F_{23}$ & 0.0075 & 3 & 1.2790 & 4 & 0.1024 & 4 & 4 \\
\hline & $F_{24}$ & 0.01389 & 1 & 1.3574 & 2 & 0.2176 & 1 & 1 \\
\hline \multirow{4}{*}{3} & $F_{31}$ & 0.0273 & 1 & 1.3759 & 1 & 0.2763 & 1 & 1 \\
\hline & $F_{32}$ & 0.0113 & 2 & 1.3644 & 2 & 0.2040 & 2 & 3 \\
\hline & $F_{33}$ & 0.001636 & 4 & 1.2914 & 4 & 0.1152 & 4 & 4 \\
\hline & $F_{34}$ & 0.0100 & 3 & 1.3312 & 3 & 0.1918 & 3 & 2 \\
\hline \multirow{6}{*}{4} & $F_{41}$ & 0.0290 & 3 & 1.3673 & 4 & 0.2799 & 3 & 1 \\
\hline & $F_{42}$ & 0.0180 & 4 & 1.3909 & 2 & 0.2384 & 4 & 4 \\
\hline & $F_{43}$ & 0.03792 & 2 & 1.4155 & 1 & 0.3130 & 2 & 2 \\
\hline & $F_{44}$ & 0.0038 & 6 & 1.322 & 5 & 0.1482 & 6 & 6 \\
\hline & $F_{45}$ & 0.051896 & 1 & 1.38098 & 3 & 0.3515 & 1 & 3 \\
\hline & $F_{46}$ & 0.00821 & 5 & 1.3208 & 6 & 0.1775 & 5 & 5 \\
\hline \multirow{4}{*}{5} & $F_{51}$ & 0.0300 & 1 & 1.3902 & 1 & 0.2892 & 1 & 1 \\
\hline & $F_{52}$ & 0.0096 & 3 & 1.3429 & 3 & 0.1926 & 3 & 3 \\
\hline & $F_{53}$ & 0.000001 & 4 & 1.2526 & 4 & 0.0680 & 4 & 4 \\
\hline & $F_{54}$ & 0.01558 & 2 & 1.3598 & 2 & 0.2262 & 2 & 2 \\
\hline \multirow{5}{*}{6} & $F_{61}$ & 0.03546 & 2 & 1.3948 & 2 & 0.3050 & 2 & 2 \\
\hline & $F_{62}$ & 0.0082 & 3 & 1.344988 & 3 & 0.18542 & 3 & 5 \\
\hline & $F_{63}$ & 0.005616 & 4 & 1.3012 & 4 & 0.1589 & 4 & 3 \\
\hline & $F_{64}$ & 0.0034 & 5 & 1.2867 & 5 & 0.1374 & 5 & 4 \\
\hline & $F_{65}$ & 0.07828 & 1 & 1.4417 & 1 & 0.4123 & 1 & 1 \\
\hline \multirow{4}{*}{7} & $F_{71}$ & 0.008728 & 3 & 1.3081 & 3 & 0.18540 & 3 & 2 \\
\hline & $F_{72}$ & 0.01482 & 1 & 1.3308 & 2 & 0.2183 & 2 & 1 \\
\hline & $F_{73}$ & 0.0008 & 4 & 1.2661 & 4 & 0.09663 & 4 & 4 \\
\hline & $F_{74}$ & 0.0102 & 2 & 1.3546 & 1 & 0.2005 & 1 & 3 \\
\hline
\end{tabular}

Real function $S: F(X) \times F(X) \rightarrow[0,1]$ is called similarity measure on $F(X)$, if $S$ satisfies the following properties:

(S1) $S\left(A, A^{c}\right)=0$ if $A$ is a crisp set.

(S2) $S(A, B)=1 \Leftrightarrow A=B$.

(S3) $S(A, B)=S(B, A)$.

(S4) For all $A, B, C \in F(X)$, if $A \subseteq B \subseteq C$, then

$$
\begin{aligned}
& S(A, C) \leq S(A, B), \\
& S(A, C) \leq S(B, C) .
\end{aligned}
$$

For instance, let $X=\left\{x_{1}, x_{2}, \ldots, x_{n}\right\}, A, B \in F(X)$; we set

$$
S(A, B)=1-\max _{x_{i} \in X}\left|\mu_{A}\left(x_{i}\right)-\mu_{B}\left(x_{i}\right)\right|
$$

then $S(A, B)$ is a similarity measure over $X$.

\section{Distance Measure of the Fuzzy Sets}

To indicate the degree of distance of two fuzzy sets, the concept of distance measure was proposed in 1992 [46].

Real function $D: F(X) \times F(X) \rightarrow[0,1]$ is called distance measure on $F(X)$, if $D$ satisfies the following properties:

(D1) $D\left(A, A^{c}\right)=1$ if $A$ is a crisp set.

(D2) $D(A, A)=0$.

(D3) $D(A, B)=D(B, A)$.

(D4) For all $A, B, C \in F(X)$, if $A \subseteq B \subseteq C$, then

$$
\begin{aligned}
& D(A, B) \leq D(A, C), \\
& D(B, C) \leq D(A, C) .
\end{aligned}
$$


TABLE 8: The global ranking order of all alternatives.

\begin{tabular}{|c|c|c|c|c|}
\hline Alternative & Opt. & Pess. & Neu. & $\begin{array}{c}\text { Lu ranking } \\
\text { order }\end{array}$ \\
\hline$F_{11}$ & 27 & 27 & 27 & 27 \\
\hline$F_{12}$ & 2 & 3 & 2 & 3 \\
\hline$F_{13}$ & 4 & 2 & 4 & 1 \\
\hline$F_{41}$ & 31 & 31 & 31 & 29 \\
\hline$F_{21}$ & 23 & 18 & 22 & 19 \\
\hline$F_{22}$ & 14 & 12 & 14 & 17 \\
\hline$F_{23}$ & 29 & 28 & 28 & 30 \\
\hline$F_{24}$ & 13 & 14 & 13 & 13 \\
\hline$F_{31}$ & 9 & 9 & 9 & 8 \\
\hline$F_{32}$ & 15 & 11 & 15 & 16 \\
\hline$F_{33}$ & 26 & 25 & 26 & 22 \\
\hline$F_{34}$ & 17 & 19 & 18 & 11 \\
\hline$F_{41}$ & 8 & 10 & 8 & 4 \\
\hline$F_{42}$ & 10 & 6 & 10 & 9 \\
\hline$F_{43}$ & 5 & 4 & 5 & 5 \\
\hline$F_{44}$ & 24 & 21 & 24 & 26 \\
\hline$F_{45}$ & 3 & 8 & 3 & 6 \\
\hline$F_{46}$ & 20 & 22 & 21 & 10 \\
\hline$F_{51}$ & 7 & 7 & 7 & 12 \\
\hline$F_{52}$ & 30 & 17 & 17 & 24 \\
\hline$F_{53}$ & 18 & 30 & 30 & 31 \\
\hline$F_{54}$ & 11 & 13 & 11 & 14 \\
\hline$F_{61}$ & 6 & 5 & 6 & 7 \\
\hline$F_{62}$ & 21 & 16 & 19 & 25 \\
\hline$F_{63}$ & 22 & 24 & 23 & 18 \\
\hline$F_{64}$ & 25 & 26 & 25 & 20 \\
\hline$F_{65}$ & 1 & 1 & 1 & 2 \\
\hline$F_{71}$ & 19 & 23 & 20 & 21 \\
\hline$F_{72}$ & 12 & 20 & 12 & 15 \\
\hline$F_{73}$ & 28 & 29 & 29 & 28 \\
\hline$F_{74}$ & 16 & 15 & 16 & 23 \\
\hline
\end{tabular}

For instance, when $\mu_{A}(x)$ and $\mu_{B}(x)$ can be integrated over the considered interval $[a, b]$, we set

$$
D(A, B)=\frac{1}{b-a} \int_{b}^{a} \frac{\left|\mu_{A}(x)-\mu_{B}(x)\right|}{\left|\mu_{A}(x)+\mu_{B}(x)\right|} d x ;
$$

then $D(A, B)$ is a distance measure over $X$.

\section{Inclusion Measure of the Fuzzy Sets}

Inclusion measure of fuzzy sets indicates the degree to which a fuzzy set is contained in another fuzzy set. In 1993, Sinha and Dougherty [47] proposed the concept as follows.

Real function $I: F(X) \times F(X) \rightarrow[0,1]$ is called inclusion measure on $F(X)$, if $I$ satisfies the following properties:

(I1) $I(X, \Phi)=0$.

(I2) $I(A, B)=1 \Leftrightarrow A \subseteq B$.
TABLE 9: The statistical numbers of linguistic variables.

\begin{tabular}{|c|c|c|c|c|c|c|c|c|}
\hline \multicolumn{2}{|c|}{ Alternative } & AS & $\mathrm{AU}$ & $S$ & $\mathrm{U}$ & VS & $\mathrm{A}$ & $\mathrm{VU}$ \\
\hline \multirow{4}{*}{1} & $F_{11}$ & & & 8 & 2 & 3 & 10 & \\
\hline & $F_{12}$ & 5 & & 6 & 2 & 6 & 4 & \\
\hline & $F_{13}$ & 1 & & 9 & & 13 & & \\
\hline & $F_{41}$ & & & 5 & 3 & 4 & 11 & \\
\hline \multirow{4}{*}{2} & $F_{21}$ & & & 10 & 2 & 4 & 7 & \\
\hline & $F_{22}$ & 1 & & 10 & 3 & 4 & 5 & \\
\hline & $F_{23}$ & & & 9 & 3 & 2 & 8 & 1 \\
\hline & $F_{24}$ & 1 & & 10 & 1 & 4 & 7 & \\
\hline \multirow{4}{*}{3} & $F_{31}$ & 3 & & 7 & 2 & 5 & 6 & \\
\hline & $F_{32}$ & & & 12 & 1 & 4 & 6 & \\
\hline & $F_{33}$ & & & 7 & 2 & 5 & 9 & \\
\hline & $F_{34}$ & 1 & & 8 & & 5 & 9 & \\
\hline \multirow{6}{*}{4} & $F_{41}$ & 4 & & 2 & 3 & 9 & 5 & \\
\hline & $F_{42}$ & & & 13 & & 5 & 5 & \\
\hline & $F_{43}$ & 2 & & 12 & & 4 & 5 & \\
\hline & $F_{44}$ & & & 10 & 3 & 3 & 7 & 1 \\
\hline & $F_{45}$ & 6 & & 4 & 2 & 4 & 6 & 1 \\
\hline & $F_{46}$ & 1 & & 6 & & 8 & 7 & 1 \\
\hline \multirow{4}{*}{5} & $F_{51}$ & & & 9 & 3 & 5 & 1 & 2 \\
\hline & $F_{52}$ & 1 & 1 & 11 & 1 & 3 & 5 & 1 \\
\hline & $F_{53}$ & & & 8 & 4 & 1 & 9 & 1 \\
\hline & $F_{54}$ & 1 & & 11 & & 5 & 4 & 2 \\
\hline \multirow{5}{*}{6} & $F_{61}$ & 3 & & 9 & 1 & 4 & 6 & \\
\hline & $F_{62}$ & & & 13 & 1 & 2 & 6 & 1 \\
\hline & $F_{63}$ & 1 & & 7 & & 5 & 9 & 1 \\
\hline & $F_{64}$ & 1 & & 6 & 1 & 6 & 7 & 2 \\
\hline & $F_{65}$ & 5 & & 9 & 1 & 4 & 4 & \\
\hline \multirow{4}{*}{7} & $F_{71}$ & 2 & & 7 & 1 & 3 & 9 & 1 \\
\hline & $F_{72}$ & 3 & 1 & 5 & 1 & 6 & 6 & 1 \\
\hline & $F_{73}$ & 1 & 1 & 5 & 2 & 5 & 8 & 1 \\
\hline & $F_{74}$ & 1 & & 10 & 4 & 3 & 5 & \\
\hline
\end{tabular}

(I3) For all $A, B, C \in F(X)$, if $A \subseteq B \subseteq C$, then

$$
\begin{aligned}
& I(C, A) \leq I(B, A), \\
& I(C, A) \leq I(C, B) .
\end{aligned}
$$

For instance, when $\mu_{A}(x)$ and $\mu_{B}(x)$ can be integrated over the considered interval $[a, b]$, we set

$$
I(A, B)=\frac{1}{b-a} \int_{b}^{a} \frac{\mu_{A}(x) \wedge \mu_{B}(x)}{\mu_{A}(x)} d x
$$

then $I(A, B)$ is an inclusion measure over $X$.

\section{Conflict of Interests}

The authors declare that there is no conflict of interests regarding the publication of this paper. 


\section{Acknowledgment}

This work was supported by National Natural Science Foundation of China (no. 61473327).

\section{References}

[1] R. E. Bellman and L. A. Zadeh, "Decision-making in a fuzzy environment," Management Science, vol. 17, no. 4, pp. 141-164, 1970.

[2] S. M. Baas and H. Kwakernaak, "Rating and ranking of multiple aspect alternatives using fuzzy sets," Automatica, vol. 13, no. 1, pp. 47-58, 1977.

[3] J. Ye, "Correlation coefficient of dual hesitant fuzzy sets and its application to multiple attribute decision making," Applied Mathematical Modelling, vol. 38, no. 2, pp. 659-666, 2014.

[4] G. Wei, X. Zhao, and R. Lin, "Some hesitant interval-valued fuzzy aggregation operators and their applications to multiple attribute decision making," Knowledge-Based Systems, vol. 46, pp. 43-53, 2013.

[5] X. Zhao, R. Lin, and G. Wei, "Hesitant triangular fuzzy information aggregation based on Einstein operations and their application to multiple attribute decision making," Expert Systems with Applications, vol. 41, no. 4, pp. 1086-1094, 2014.

[6] B. Farhadinia, "A novel method of ranking hesitant fuzzy values for multiple attribute decision-making problems," International Journal of Intelligent Systems, vol. 28, no. 8, pp. 752-767, 2013.

[7] T.-C. Wang and T.-H. Chang, "Application of TOPSIS in evaluating initial training aircraft under a fuzzy environment," Expert Systems with Applications, vol. 33, no. 4, pp. 870-880, 2007.

[8] D. Tadić, A. Aleksić, M. Stefanović, and S. Arsovski, "Evaluation and ranking of organizational resilience factors by using a twostep fuzzy AHP and fuzzy TOPSIS," Mathematical Problems in Engineering, vol. 2014, Article ID 418085, 13 pages, 2014.

[9] P. Liu and Y. Wang, "Multiple attribute decision-making method based on single-valued neutrosophic normalized weighted Bonferroni mean," Neural Computing and Applications, vol. 25, no. 7-8, pp. 2001-2010, 2014.

[10] L. Huchen, L. Qinglian, and W. Jing, "Dependent interval 2tuple linguistic aggregation operators and their application to multiple attribute group decision making," International Journal of Uncertainty, Fuzziness and Knowledge-Based Systems, vol. 22, no. 5, pp. 717-735, 2014.

[11] M. Xia and Z. Xu, "Group decision making based on intuitionistic multiplicative aggregation operators," Applied Mathematical Modelling, vol. 37, no. 7, pp. 5120-5133, 2013.

[12] M. Xia, Z. Xu, and H. Liao, "Preference relations based on intuitionistic multiplicative information," IEEE Transactions on Fuzzy Systems, vol. 21, no. 1, pp. 113-133, 2013.

[13] L.-H. Chen and C.-C. Tu, "Time-validating-based Atanassov's intuitionistic fuzzy decision making," IEEE Transactions on Fuzzy Systems, vol. 23, no. 4, pp. 743-756, 2015.

[14] A. Hadi-Vencheh and M. Mirjaberi, "Fuzzy inferior ratio method for multiple attribute decision making problems," Information Sciences, vol. 277, pp. 263-272, 2014.

[15] X. Zhang and Z. Xu, "Extension of TOPSIS to multiple criteria decision making with pythagorean fuzzy sets," International Journal of Intelligent Systems, vol. 29, no. 12, pp. 1061-1078, 2014.

[16] L. Anojkumar, M. Ilangkumaran, and V. Sasirekha, "Comparative analysis of MCDM methods for pipe material selection in sugar industry," Expert Systems with Applications, vol. 41, no. 6, pp. 2964-2980, 2014.

[17] H. J. Zimmermann, Fuzzy Set, Decision Making and Expert System, Kluwer Academic Publishers, Boston, Mass, USA, 1987.

[18] C. Wei and X. Tang, "An intuitionistic fuzzy group decisionmaking approach based on entropy and similarity measures," International Journal of Information Technology and Decision Making, vol. 10, no. 6, pp. 1111-1130, 2011.

[19] X. Qi, C. Liang, and J. Zhang, "Generalized cross-entropy based group decision making with unknown expert and attribute weights under interval-valued intuitionistic fuzzy environment," Computers and Industrial Engineering, vol. 79, pp. 52-64, 2015.

[20] Z. Xu and M. Xia, "Hesitant fuzzy entropy and cross-entropy and their use in multiattribute decision-making," International Journal of Intelligent Systems, vol. 27, no. 9, pp. 799-822, 2012.

[21] P. Luukka, "Feature selection using fuzzy entropy measures with similarity classifier," Expert Systems with Applications, vol. 38, no. 4, pp. 4600-4607, 2011.

[22] F. H. Lotfi and R. Fallahnejad, "Imprecise shannon's entropy and multi attribute decision making," Entropy, vol. 12, no. 1, pp. 53$62,2010$.

[23] X.-Z. Wang and C.-R. Dong, "Improving generalization of fuzzy IF-THEN rules by maximizing fuzzy entropy," IEEE Transactions on Fuzzy Systems, vol. 17, no. 3, pp. 556-567, 2009.

[24] K. Yao, "Sine entropy of uncertain set and its applications," Applied Soft Computing, vol. 22, pp. 432-442, 2014.

[25] F. Jin, L. Pei, H. Chen, and L. Zhou, "Interval-valued intuitionistic fuzzy continuous weighted entropy and its application to multi-criteria fuzzy group decision making," Knowledge-Based Systems, vol. 59, pp. 132-141, 2014.

[26] Y. Zhang, P. Li, Y. Wang, P. Ma, and X. Su, "Multiattribute decision making based on entropy under interval-valued intuitionistic fuzzy environment," Mathematical Problems in Engineering, vol. 2013, Article ID 526871, 8 pages, 2013.

[27] J. Fan and W. Xie, "Distance measure and induced fuzzy entropy," Fuzzy Sets and Systems, vol. 104, no. 2, pp. 305-314, 1999.

[28] C.-P. Wei, P. Wang, and Y.-Z. Zhang, "Entropy, similarity measure of interval-valued intuitionistic fuzzy sets and their applications," Information Sciences, vol. 181, no. 19, pp. 42734286, 2011.

[29] Z. Qiansheng and J. Shengyi, "Relationships between entropy and similarity measure of interval-valued intuitionistic fuzzy sets," International Journal of Intelligent Systems, vol. 25, pp. 1121-1140, 2010.

[30] C.-M. Hwang, M.-S. Yang, and W.-L. Hung, "On similarity, inclusion measure and entropy between type-2 fuzzy sets," International Journal of Uncertainty, Fuzziness and KnowledgeBased Systems, vol. 20, no. 3, pp. 433-449, 2012.

[31] M.-S. Yang and D.-C. Lin, "On similarity and inclusion measures between type-2 fuzzy sets with an application to clustering," Computers and Mathematics with Applications, vol. 57, no. 6, pp. 896-907, 2009.

[32] C.-M. Hwang, M.-S. Yang, W.-L. Hung, and E. Stanley Lee, "Similarity, inclusion and entropy measures between type-2 fuzzy sets based on the Sugeno integral," Mathematical and Computer Modelling, vol. 53, no. 9-10, pp. 1788-1797, 2011.

[33] D. Dubois and H. Prade, Fuzzy Sets and Systems, Theory and Applications, Academic Press, New York, NY, USA, 1980. 
[34] D. Dubois and H. Prade, "Operations on fuzzy numbers," International Journal of Systems Science, vol. 9, no. 6, pp. 626631, 1978.

[35] G. J. Klir, Uncertainty and Information, Foundations of Generalized Information Theory, Wiley-Interscience, New York, NY, USA, 2006.

[36] J. Qin and X. Liu, "Multi-attribute group decision making using combined ranking value under interval type-2 fuzzy environment," Information Sciences, vol. 297, pp. 293-315, 2015.

[37] Y. Jun, "Multiple attribute decision-making method based on the possibility degree ranking method and ordered weighted aggregation operators of interval neutrosophic numbers," Journal of Intelligent \& Fuzzy Systems, vol. 28, no. 3, pp. 1307-1317, 2015.

[38] F. Lolli, A. Ishizaka, R. Gamberini, B. Rimini, and M. Messori, "FlowSort-GDSS-a novel group multi-criteria decision support system for sorting problems with application to FMEA," Expert Systems with Applications, vol. 42, no. 17-18, pp. 63426349, 2015.

[39] C. E. Shannon, "A mathematical theory of communication," The Bell System Technical Journal, vol. 27, no. 3, pp. 379-423, 1948.

[40] F. Kong, Fuzzy Multiple Attribute Decision Making Theory, Methods and Its Applications, Chinese Agricultural Science and Technology Press, 2008, (Chinese).

[41] S. Yiying and Y. Xuehai, "A possibility-based method for ranking fuzzy numbers and applications to decision making," Journal of Intelligent and Fuzzy Systems, vol. 29, pp. 337-349, 2015.

[42] V.-N. Huynh, Y. Nakamori, and J. Lawry, "A probability-based approach to comparison of fuzzy numbers and applications to target-oriented decision making," IEEE Transactions on Fuzzy Systems, vol. 16, no. 2, pp. 371-387, 2008.

[43] S.-T. Lu, S.-H. Yu, and D.-S. Chang, "Using fuzzy multiple criteria decision-making approach for assessing the risk of railway reconstruction project in Taiwan," The Scientific World Journal, vol. 2014, Article ID 239793, 14 pages, 2014.

[44] A. D. Luca and S. Termini, "A definition of a nonprobabilistic entropy in the setting of fuzzy sets theory," Information and Control, vol. 20, no. 4, pp. 301-312, 1972.

[45] C. P. Pappis and N. I. Karacapilidis, "A comparative assessment of measures of similarity of fuzzy values," Fuzzy Sets and Systems, vol. 56, no. 2, pp. 171-174, 1993.

[46] L. Xuecheng, "Entropy, distance measure and similarity measure of fuzzy sets and their relations," Fuzzy Sets and Systems, vol. 52, no. 3, pp. 305-318, 1992.

[47] D. Sinha and E. R. Dougherty, "Fuzzification of set inclusion: theory and applications," Fuzzy Sets and Systems, vol. 55, no. 1, pp. 15-42, 1993. 


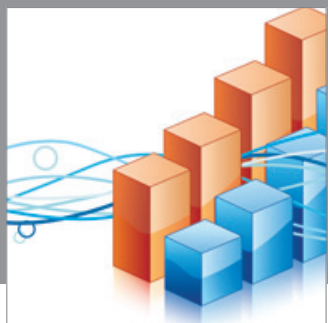

Advances in

Operations Research

mansans

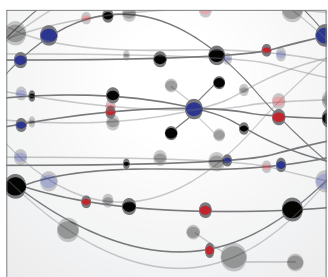

The Scientific World Journal
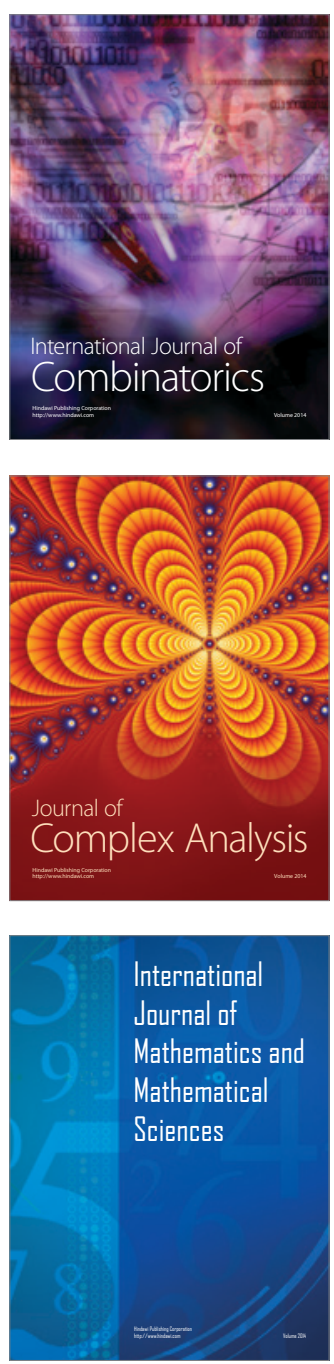
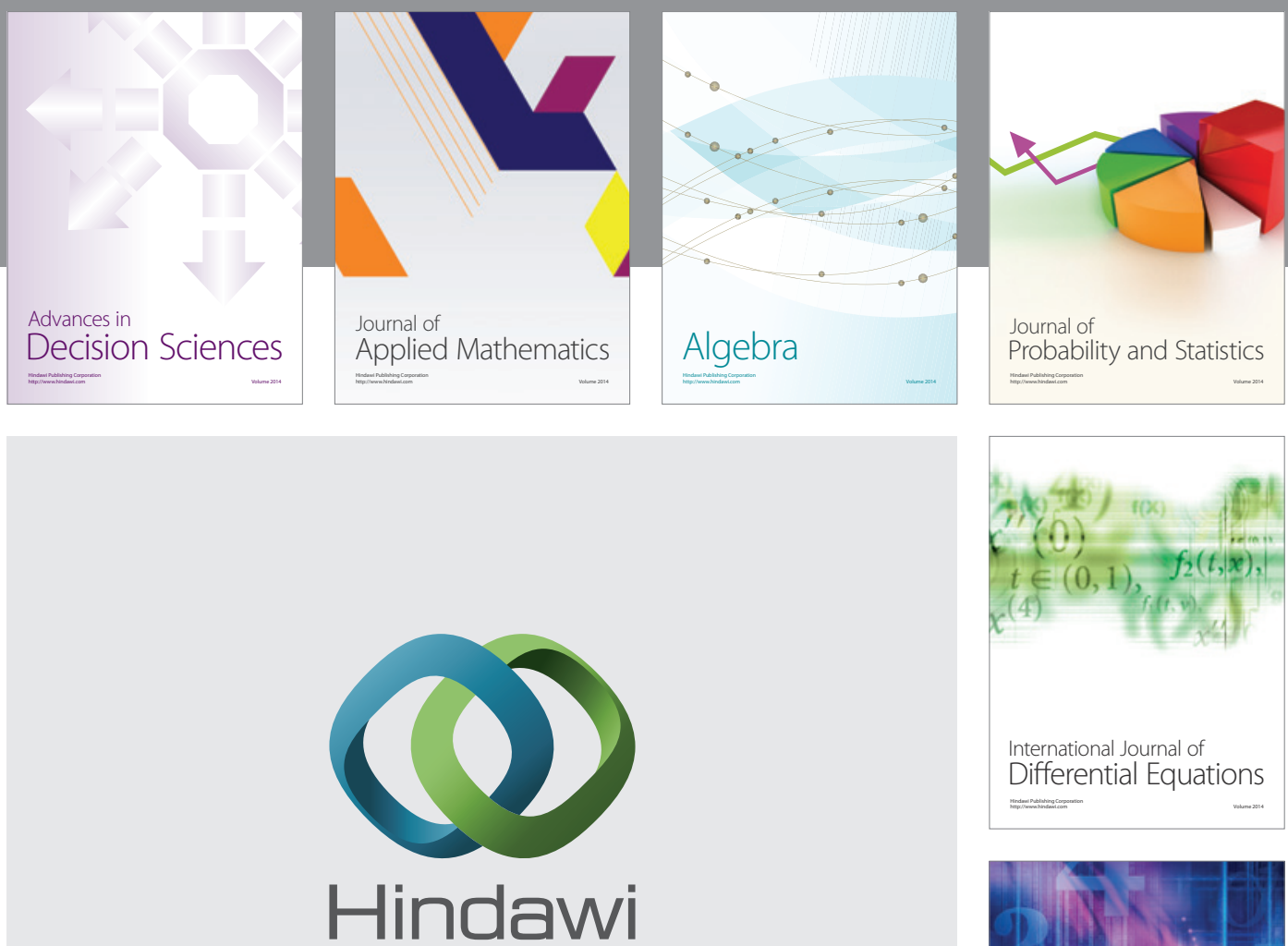

Submit your manuscripts at http://www.hindawi.com
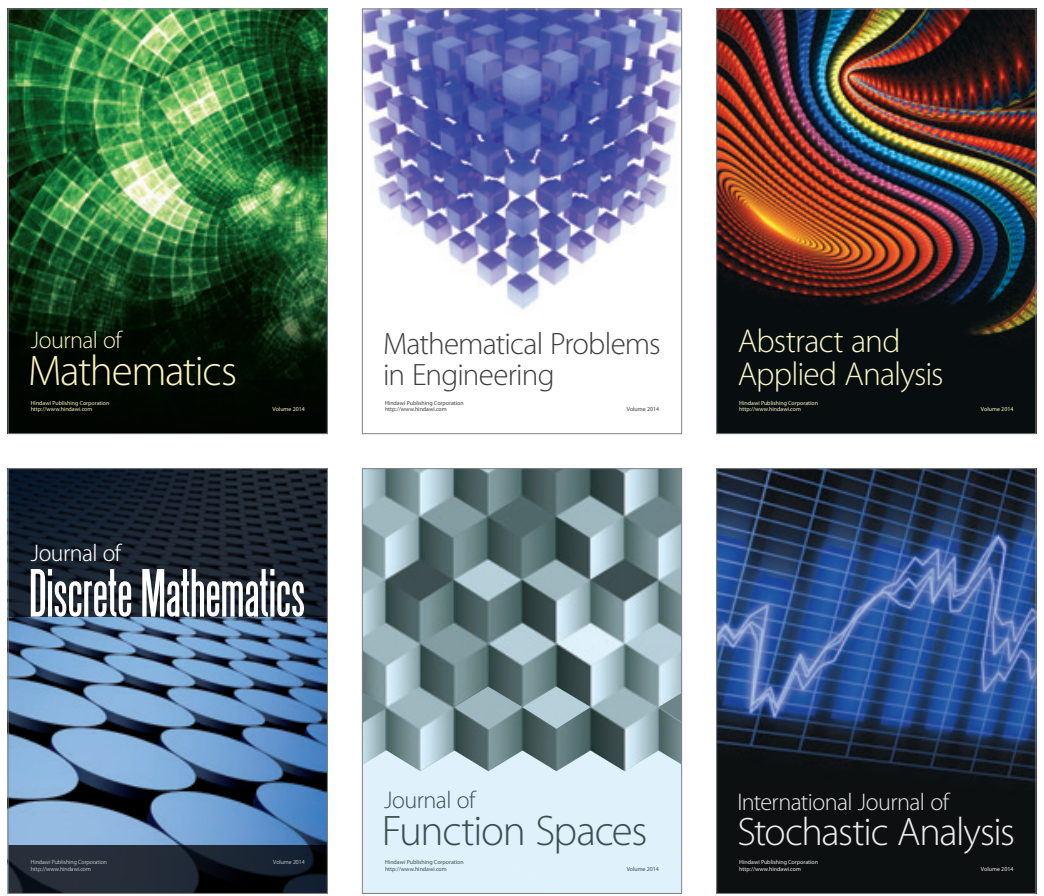

Journal of

Function Spaces

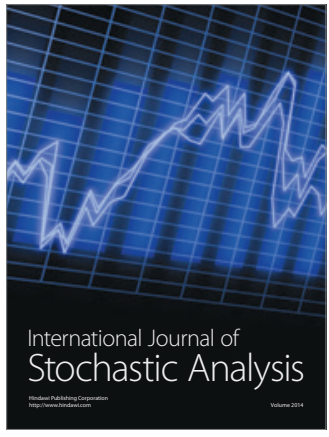

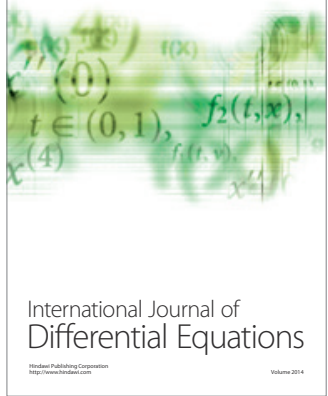
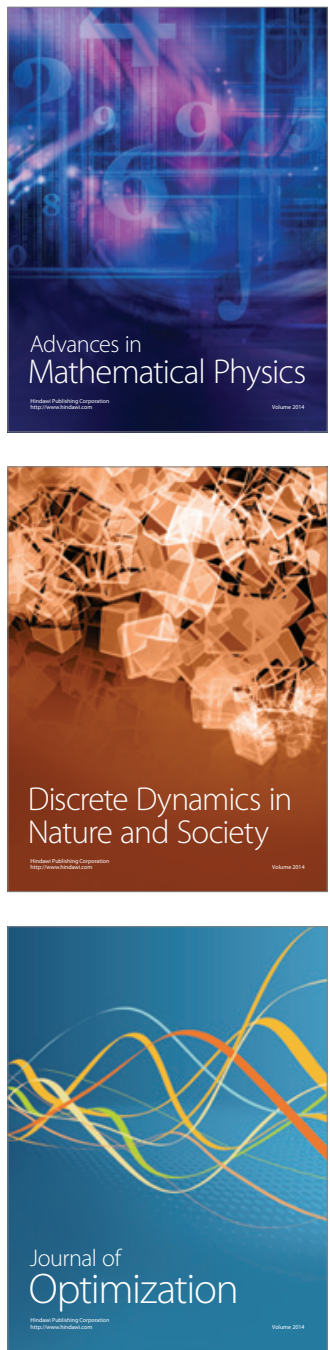